2013s-14

\title{
Disentangling Continuous Volatility from Jumps in Long-Run Risk-Return Relationships
}

\author{
Eric Jacquier, Cédric Okou
}

\begin{tabular}{c}
\hline Série Scientifique \\
Scientific Series
\end{tabular}

\author{
Montréal \\ Juin 2013
}

(C) 2013 Eric Jacquier, Cédric Okou. Tous droits réservés. All rights reserved. Reproduction partielle permise avec citation du document source, incluant la notice $\mathbb{C}$.

Short sections may be quoted without explicit permission, if full credit, including (C) notice, is given to the source.
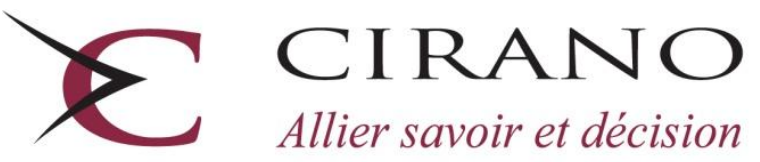

Allier savoir et décision

Centre interuniversitaire de recherche en analyse des organisations 


\section{CIRANO}

Le CIRANO est un organisme sans but lucratif constitué en vertu de la Loi des compagnies du Québec. Le financement de son infrastructure et de ses activités de recherche provient des cotisations de ses organisations-membres, d'une subvention d'infrastructure du Ministère du Développement économique et régional et de la Recherche, de même que des subventions et mandats obtenus par ses équipes de recherche.

CIRANO is a private non-profit organization incorporated under the Québec Companies Act. Its infrastructure and research activities are funded through fees paid by member organizations, an infrastructure grant from the Ministère du Développement économique et régional et de la Recherche, and grants and research mandates obtained by its research teams.

\section{Les partenaires du CIRANO}

\section{Partenaire majeur}

Ministère de l'Enseignement supérieur, de la Recherche, de la Science et de la Technologie

Partenaires corporatifs

Autorité des marchés financiers

Banque de développement du Canada

Banque du Canada

Banque Laurentienne du Canada

Banque Nationale du Canada

Banque Scotia

Bell Canada

BMO Groupe financier

Caisse de dépôt et placement du Québec

Fédération des caisses Desjardins du Québec

Financière Sun Life, Québec

Gaz Métro

Hydro-Québec

Industrie Canada

Investissements PSP

Ministère des Finances et de l'Économie

Power Corporation du Canada

Rio Tinto Alcan

State Street Global Advisors

Transat A.T.

Ville de Montréal

\section{Partenaires universitaires}

École Polytechnique de Montréal

École de technologie supérieure (ÉTS)

HEC Montréal

Institut national de la recherche scientifique (INRS)

McGill University

Université Concordia

Université de Montréal

Université de Sherbrooke

Université du Québec

Université du Québec à Montréal

Université Laval

Le CIRANO collabore avec de nombreux centres et chaires de recherche universitaires dont on peut consulter la liste sur son site web.

Les cahiers de la série scientifique (CS) visent à rendre accessibles des résultats de recherche effectuée au CIRANO afin de susciter échanges et commentaires. Ces cahiers sont écrits dans le style des publications scientifiques. Les idées et les opinions émises sont sous l'unique responsabilité des auteurs et ne représentent pas nécessairement les positions du CIRANO ou de ses partenaires.

This paper presents research carried out at CIRANO and aims at encouraging discussion and comment. The observations and viewpoints expressed are the sole responsibility of the authors. They do not necessarily represent positions of CIRANO or its partners. 


\title{
Disentangling Continuous Volatility from Jumps in Long- Run Risk-Return Relationships
}

\author{
Eric Jacquier ${ }^{\dagger}$, Cédric Okou
}

\begin{abstract}
Résumé / Abstract
Realized variance can be broken down into continuous volatility and jumps. We show that these two components have very different predictive powers on future long-term excess stock market returns. While continuous volatility is a key driver of medium to long-term risk-return relationships, jumps do not predict future medium- to long-term excess returns. We use inference methods robust to persistent predictors in a multi-horizon setup. That is, we use a rescaled Student-t to test for significant risk-return links, give asymptotic arguments and simulate its exact behavior under the null in the case of multiple regressors with different degrees of persistence. Then, with Wald tests of equality of the risk-return relationship at multiple horizons, we find no evidence against a proportional relationship, constant across horizons, between long-term continuous volatility and future returns. Two by-products of our analysis are that imposing model-based constraints on long term regressions can improve their efficiency, and short-run estimates are sensitive to short-term variability of the predictors.
\end{abstract}

Mots clés/keywords : predictability, realized variance, continuous volatility, jumps, long-run returns, persistent regressor.

\footnotetext{
* The paper has benefited from the comments of an anonymous referee and the editor George Tauchen, and René Garcia, Benoit Perron, Jin Duan, Debbie Lucas, Tolga Cenesizoglu, Aurelio Vasquez, Januj Juneja, seminar participants at HEC Montréal, Bank of Canada, the 2011 meetings of the Financial Management Association and the Northern Finance Association, the 2011 international Conference on Computing in Economics and Finance, the 2011 North American Summer Meeting of the Econometric Society, the 2010 Mathematical Finance Days, the 2010 meetings of the Canadian Economic Association and the 2010 MITACS/CORS Annual Conference. We acknowledge financial support from the Institut de Finance Mathematique de Montréal, the HEC professorship in Derivative Securities and the MIT-Sloan research funds.

${ }^{\dagger}$ HEC Montreal and MIT Sloan.

ॠUniversity of Quebec at Montreal, okou.cedric@uqam.ca.
} 
The predictability of stock returns is a fundamental topic in empirical asset pricing. Since the initial work of Fama and French (1988), many papers have presented evidence of predictability in long-term excess returns. Others express some healthy degree of skepticism, as for example Boudoukh et al. (2008) and Goyal and Welch (2008). In the literature that doubts predictability, a running theme is that many of the predictors result from data mining. In sharp contrast, expected future volatility is a prime candidate predictor of future returns for economic reasons provided by numerous asset pricing models of the link between risk and return. See for example Merton (1971, 1980) and Bansal and Yaron (2004). Lettau and Ludvigson (2010) report strong evidence that conditional expected volatility can predict future returns. Bandi and Perron (2008), hereafter BP, find that realized market variance can be a stronger predictor of long-term future excess returns than the dividend yield or the consumption-to-wealth ratio.

Realized variance, as used by BP, has tremendous appeal as an estimator of the unobservable variance, see for example Andersen, Bollerslev, Diebold and Labys (2003). However, in the presence of jumps in returns, realized variance contains both a continuous volatility and a jump components, see, Andersen, Bollerslev and Diebold (2010) for a survey. Huang and Tauchen (2005) find that jumps are not negligible, accounting for seven percent of the stock market daily variance. Thus, jump-diffusion models are now widely used to describe the stock returns dynamics. Further, jump risk is now a fundamental premise of the option pricing literature, see for example Johannes (2004), Eraker, Johannes and Polson (2003), Carr and Wu (2003a,b), Bates (2000), Kim, Oh and Brooks (1994). If jump risk is priced in options, one would expect it to be priced in the underlying returns themselves. Back (1991) shows that jump risk will be priced if the state price density jumps simultaneously with the security. Yan (2011) proposes a model where stocks earn a premium for systematic jumps that are more negatively correlated with jumps in the stochastic discount factor. On the other hand, jump risk would not be priced if one assumes, as in Merton (1976), that the jumps of individual securities diversify cross-sectionally, because these sharp discontinuities in individual stocks prices may be unsystematic. The impact of past jump risk on future long-term risk premiums remains an open question.

Continuous volatility and jumps do display distinct features. For instance, Andersen et 
al. (2007) show that continuous volatility is more persistent than the jumps, and disentangling these two components improves the out-of-sample volatility forecasts. If disentangling jumps from continuous volatility results in better volatility forecasts, it is natural to ask whether these "better" forecasts can lead to better forecasts of future returns. Bollerslev and Todorov (2010) disentangle systematic diffusive and jump risks (betas). Using a large cross-section of stocks, they show that jump betas are typically larger but less persistent than diffusion betas. The implication of this result on whether there is a premium for jump risk is unclear. Thus far, there is little evidence of whether jumps allow to predict the risk premium, especially for the longer term.

In this article, we investigate whether disentangling the market index continuous volatility and jumps allows to better predict future excess return at different horizons. Namely, we separate continuous volatility from jumps before analyzing the multi-horizon risk vs. return trade-offs. We rely on a nonparametric decomposition of the realized variance as in Huang and Tauchen (2005). ${ }^{1}$

The contribution of this paper is two-fold. First, we explore the predictive power of the continuous and jump components of realized variance in forecasting long-term market excess return. We focus on the term structures of risk-return trade-offs for these two components, as they may have different implications on the pricing and hedging of long-horizon derivatives, or on asset allocation. Our empirical results indicate that medium- to long-term market excess returns are strongly related to past continuous volatility. In contrast, we do not find a significant link between long-term market excess returns and past jumps. This is consistent with the view that jumps intrinsically characterize short term dynamics. Continuous volatility is the key driver of risk-return trade-offs at medium to long horizons, consistent with evidence as in Jarrow and Rosenfeld (1984).

Second, the paper performs equality restrictions tests on the term structure of risk-return trade-offs, after extracting jumps from the past market realized variance. This allows us to explore a possible horizon effect. To do this, we develop a normalized version of the classical Wald test statistic. We derive its asymptotic distribution and provide the necessary simulated critical values.

\footnotetext{
${ }^{1}$ A thorough justification for a model-free decomposition of realized variance can be found in Aït-Sahalia (2004), Barndorff-Nielsen and Shephard $(2004,2006)$. Other nonparametric jump detection methods are discussed in Andersen, Dobrev and Schaumburg (2012), Lee and Hanning (2010), Aït-Sahalia and Jacod (2009), Lee and Mykland (2008), Jiang and Oomen (2005).
} 
Our inference explicitly accounts for persistence in the predictor variable in a multi-horizon setup. The empirical evidence is consistent with a flat term structure of trade-offs between continuous volatility and future market excess returns.

This paper is also related to the literature on intertemporal capital asset pricing models (ICAPM). Scruggs (1998) successfully uses a two-factor ICAPM to analyze hedging demand requirements. Bali and Engle (2010) estimate an intertemporal CAPM using stocks and equity portfolios. Looking at possible shifts in investment opportunities, Campbell and Viceira (2005) characterize the term structure of risk-return trade-offs. Bollerslev, Tauchen and Zhou (2009) argue that the variance risk premium can substantially account for the time variation in future excess returns at long horizons. Bollerslev, Sizova and Tauchen (2012) build on the long-run risk framework of Bansal and Yaron (2004) to develop an equilibrium model which accounts for volatility asymmetries and dependencies. However, while these papers explore intertemporal risk-return relationships, they do not distinguish between jump and diffusion risk across different horizons. We provide general inference methods and new insights about the term structures of jump and diffusion risk-return trade-offs.

The remainder of this paper is as follows. Section 1 presents estimators for total quadratic variation, continuous volatility and jumps. Section 2 describes the data. Section 3 discusses estimation results, inference and simulated critical values for the rescaled $t$-statistic. Section 4 proposes a specification linking market excess return only to past market continuous volatility. Relying on a normalized version of the Wald test statistic, denoted the rescaled Wald statistic, we test the equality of slope coefficients over different horizons. Section 5 concludes.

\section{Realized Variance, Continuous Volatility and Jumps}

In this section, we introduce the estimators to be used in lieu of the unknown latent variables to estimate the relationship:

$$
R_{t, t+h}=a_{h}+b_{h} C_{t-h, t}+\gamma_{h} \mathcal{J}_{t-h, t}^{2}+e_{t, t+h}
$$


where $R_{t, t+h}$ denotes the market log-excess return over months $t+1$ to $t+h, C_{t-h, t}$ and $\mathcal{J}_{t-h, t}^{2}$ are the continuous volatility and the jump components over months $t-h$ to $t$, and $e_{t, t+h}$ is the prediction error. We will run these regressions for $h$ varying from 1 to 120 months. The no-intercept version of Eqn.(1) is consistent with risk vs. return models as in Merton (1980). It is therefore preferable for estimation efficiency, see Campbell and Thompson (2008). We run both the intercept and the no-intercept versions of Eqn. (1).

\section{$1.1 \quad$ Realized Variance}

The continuous volatility $C_{t-h, t}$ and the jump component $\mathcal{J}_{t-h, t}^{2}$ are embedded in the quadratic variation. So, we first introduce realized variance, the estimator of quadratic variation. Say that month $t$ contains $n_{t}$ daily returns denoted $r_{t, i}$, where $r_{t, 1}$ and $r_{t, n_{t}}$ are the returns on the first and last days of the month. Similarly, we write $h(t)$, the set of $h$ months, $t-h+1$ to $t$, and $n_{h(t)}$, the number of days in these $h$ months. Realized variance (RV) is a simple and consistent estimator of the second-order path variation of the log-returns, aka quadratic variation. RV over a given time interval is the sum of squared high frequency returns over that interval. With daily returns as high frequency returns, and a h-month interval, the h-month realized variance is therefore

$$
R V_{t-h, t}=\sum_{i=1}^{n_{h(t)}} r_{h(t), i}^{2}
$$

For a given interval, as the sampling frequency increases, RV is a consistent estimator of the quadratic variation of the $\log$-price process $\log \left(p_{t}\right)$, over this interval. In the presence of infrequent jumps, the quadratic variation [.] of $\log \left(p_{t}\right)$, contains two elements, the integrated continuous variance and the discontinuous squared jumps process:

$$
[\log p]_{t-h, t}=\int_{t-h}^{t} \sigma_{s}^{2} d s+\sum_{t-h \leq s \leq t}\left(\log \left(p_{s}\right)-\log \left(p_{s-}\right)\right)^{2},
$$

with $\log \left(p_{s-}\right)=\lim _{\epsilon \downarrow 0} \log \left(p_{s-\epsilon}\right)$. We now discuss how to estimate these two components. 


\subsection{Disentangling Jumps and Continuous Volatility}

Barndorff-Nielsen and Shephard (2004, 2006), hereafter BNS, provide theoretical and empirical motivation for the bipower variation as an estimator robust to jumps of the integrated continuous volatility, the first term on the right-hand side of Eqn.(3). ${ }^{2}$ The bipower variation (BV) is a scaled sum of cross-products of adjacent absolute returns. Tauchen and Zhou (2011) expand the use of $\mathrm{BV}$ to a formal jump detection framework, testing for significant jumps and estimating parameters such as the jump intensity, mean and variance.

The h-month bipower variation, using the daily returns as high-frequency returns is then:

$$
B V_{t-h, t}=\frac{\pi}{2} \frac{n_{h(t)}}{n_{h(t)}-1} \sum_{i=2}^{n_{h(t)}}\left|r_{h(t), i-1}\right|\left|r_{h(t), i}\right|
$$

BNS (2004) show that a consistent estimator of jumps is simply the difference between the realized variance and the bipower variation. Namely,

$$
\lim _{n_{h(t)} \rightarrow \infty}\left(R V_{t-h, t}-B V_{t-h, t}\right)=\sum_{t-h+1 \leq s \leq t} \mathcal{J}_{s}^{2}
$$

While the difference in the left hand side of Eqn.(5) converges asymptotically to a non negative quantity, it can however be negative for a given sample. Therefore, it can not be used in this simple form. Huang and Tauchen (2005) design a significance test of the jump based on the relative difference

$$
R J_{t-h, t}=\frac{R V_{t-h, t}-B V_{t-h, t}}{R V_{t-h, t}}
$$

Specifically, they compute the studentized relative difference

$$
z_{t-h, t}=\frac{R J_{t-h, t}}{\sqrt{\frac{\left(\frac{\pi}{2}\right)^{2}+\pi-5}{n_{h(t)}} \max \left(1, \frac{T P_{t-h, t}}{B V_{t-h, t}^{2}}\right)}}
$$

where $T P_{t-h, t}$ is the tripower quarticity, a consistent estimator of the integrated quarticity. The

\footnotetext{
${ }^{2}$ We obtain similar empirical results with the MedRV estimator of integrated variance proposed by Andersen, Dobrev and Schaumburg (2012).
} 
integrated quarticity is the volatility of realized volatility. The multi-month tripower quarticity is

$$
T P_{t-h, t}=\mu_{4 / 3}^{-3} \frac{n_{h(t)}^{2}}{n_{h(t)}-2} \sum_{i=3}^{n_{h(t)}}\left|r_{h(t), i-2}\right|^{4 / 3}\left|r_{h(t), i-1}\right|^{4 / 3}\left|r_{h(t), i}\right|^{4 / 3}
$$

where $\mu_{4 / 3}=2^{2 / 3} \Gamma\left(\frac{7}{6}\right) / \Gamma\left(\frac{1}{2}\right)$. Hang and Tauchen show that $z_{t}$, the studentized relative difference between $R V$ and $B V$ is asymptotically a unit normal distribution. Their Monte Carlo simulations indicate that the normal distribution is a good approximation of $z_{t}$, with good size and power properties.

The possible negativity of $R V-B V$ in small sample, is addressed as follows. Andersen, Bollerslev, and Diebold (2007) compute the significant jump, a shrinkage estimator based on a chosen $x \%$ confidence level of $z_{t}$. The significant jump is

$$
\mathcal{J}_{t-h, t}^{2}(x)=I\left(z_{t-h, t}>\zeta_{x}\right)\left[R V_{t-h, t}-B V_{t-h, t}\right]
$$

where $I(\cdot)$ is an indicator function and $\zeta_{x}$ is the normal critical value for the $x \%$ confidence level. The indicator guarantees the non-negativity of the significant jump estimator, coherently with a significance level chosen for $z$. Note that when $x=50 \%$, due to the standard properties of the Gaussian distribution, the significant jump in Eqn.(9) is simply $\max \left[R V_{t-h, t}-B V_{t-h, t}, 0\right]$, initially suggested to address the negativity issue in the early literature.

From Eqn.(9), we can estimate the continuous component of the quadratic variation as:

$$
C_{t-h, t}(x)=I\left(z_{t-h, t} \leq \zeta_{x}\right) R V_{t-h, t}+I\left(z_{t-h, t}>\zeta_{x}\right) B V_{t-h, t}
$$

From hereon, we will refer to $C_{t-h, t}$ as the continuous volatility. There is discussion in the literature that realized volatility measures may be contaminated by high frequency market microstructure noise, see Andersen, Bollerslev and Diebold (2010) for a survey. The impact here should be minimal as our high frequency is not intra-day, but daily. 


\section{Data}

We use the NYSE / AMEX value-weighted index with dividends as the market proxy from January 2, 1952 to December 31, 2009. We obtained similar results with the S\&P 500. For the risk-free rate, we use the 30-day T-bill rate. Daily series are obtained from the CRSP database. We compute the h-month continuously compounded excess return $R_{t, t+h}$ by summing up the daily excess returns over the months $t+1$ to $t+h$.

For a given horizon $h$, regression (1) will be run with monthly observations of the h-month left-hand side variable $R_{t, t+h}$, and the estimates $\mathcal{J}_{t-h, t}^{2}$, and $C_{t-h, t}$ of the unknown latent variables in the right-hand side. This induces overlapping errors and their ensuing serial dependence, which we will account for in the analysis. The efficiency gains of using overlapping returns despite the related complication have been well known since Hansen and Hodrick (1980) seminal paper.

The high-frequency data in our analysis is daily, leading to about 22 observations per month. However, the convergence of second order variation estimator is based upon limiting arguments of increasingly finely sampled returns. This is commonly used in the framework of intraday data, but an analogous asymptotics can be argued for in long-run analysis where the daily records constitute the high frequency. French, Schwert and Stambaugh (1987) find evidence of a positive relation between monthly expected risk premium and variance, using daily returns to estimate monthly variance.

Table 1 presents descriptive statistics for the monthly market excess return, and the onemonth realized variance $R V$, continuous sample path volatility $C$, and squared jumps $\mathcal{J}^{2}$. The averages of these monthly series indicate that the significant jumps make a bit less than $10 \%$ of the total quadratic variation. This is consistent with the possibility of significant but infrequent jumps in the sample path. Higher moments of $R V, C$ and $\mathcal{J}^{2}$ series confirm their well-established rightskewed, fat-tailed distributions. The summary statistics show important asymmetry and kurtosis for the second order variation series as compared to the distribution of excess returns. Andersen, Bollerslev and Diebold (2007) document similar empirical regularities for the daily S\&P 500 index from January 1990 through December 2002. Table 1 also reports the first-order autocorrelations 
for the full sample and two subsamples chosen to exclude 1987 and the post-2007 financial crash. One could believe that crisis-related spikes in returns would "break" the dependence structure and thus, largely decrease the persistence in second order variation. It appears not to be the case as the subsample autocorrelations are only slightly lower than the full sample ones.

The goal of our paper is the estimation of the predictive power (of the components) of multiperiod realized variance on multi-period excess returns. This is different from the well explored question of the ability of realized variance to predict itself. Andersen, Bollerslev and Meddahi (2004) document the ability of past realized variance to predict future realized variance at short horizons. Andersen, Bollerslev and Diebold (2007) regress realized variance on past values of itself and past jumps, for horizons of 1 day, 1 week and 1 month. Their results show that jumps do not have marginal predictive power over volatility beyond a week. Our horizons are far longer than most of these studies, and so is our high frequency which is daily. It is therefore interesting to compare stylized facts from these existing studies with our own constructs and horizons. We regress realized variance on past continuous volatility and jumps for horizons of 1 to 120 months. Our results are consistent with theirs: we detect no ability of past jumps to predict future realized variance. Table 2 shows the regression of the logarithms of realized variance on past values of the logarithm of continuous volatility. The slope coefficient is statistically significant at a conventional $5 \%$ level until a 3-year horizon. Beyond, both t-statistic and R-square collapse. This is consistent with a strongly autocorrelated but stationary volatility.

Figure 1 plots the monthly market excess returns from January 1952 to December 2009. Among these 696 monthly returns, we identify 73 significant jumps using the jump test statistic $z_{t}$ at a $95 \%$ confidence level. Figure 2 plots the monthly realized variance (top panel) in logarithm scale, and squared jumps (middle panel). The jumps seem to contribute a lot to quadratic variation around the October 1987 crash and the 2008 financial crisis. However, the middle panel reveals two other interesting facts. First, jump activity is much higher after 1980 than before, independently of these crisis periods. Second, there is no evidence that jump activity is generally related to the level of realized variance. To verify this, the third (bottom) panel of Figure 1 is a scatter plot of continuous volatility vs. realized volatility. The 45 degree line shows all the months with no 
detected jump activity. Strikingly enough, this graph shows no evidence that months with higher realized volatility are also months with more jump activity. To the contrary, months with jumps, i.e. when $C_{t}$ is less than $R V_{t}$, seem to occur randomly through the whole range of $R V_{t}$ values.

As our high-frequency data are only daily, one may worry about the quality of the asymptotic approximation for the test-statistic $z_{t}$ presented in the previous section. We therefore need to verify, for our sampling scheme, the results in Huang and Tauchen (2005). Figure 3 is a normal probability plot of the estimates of the jump test statistic $z_{t}$, computed over the sample. It provides a visual confirmation that the normal distribution is a good approximation for this statistic.

Finally, Figure 4 presents the autocorrelation function of $R V_{t}$, as well as $C_{t}$ and $\mathcal{J}_{t}^{2}$ obtained with a $95 \%$ jump significance level. The top panel confirms that $R V_{t}$ has high autocorrelations decaying slowly. The bottom panel shows that, in contrast, $\mathcal{J}_{t}^{2}$ has little or no persistence. This is consistent with the view that the jump component captures occasional discontinuities in the total quadratic variation. In contrast, Panel $\mathrm{b}$ reveals that the autocorrelation of the one-month continuous volatility $C_{t}$ is also high and tapers off only gradually. The autocorrelation of the continuous volatility may be sensitive to the significance level used to compute $C_{t}$ and $\mathcal{J}_{t}^{2}$. Therefore we conduct a sensitivity analysis to the significance level. Figure 5 shows the ACF of the continuous volatility - panels (a-c), and jumps - panel (d), for significance levels 50\%, 90\% and 99\%. There are no notable differences in these ACFs. Thus, the dependence structure of the continuous volatility and jump components is robust to the choice of the significance level.

\section{Estimation}

\subsection{Risk-Return Trade-Off with Continuous Volatility Disentangled from Jumps}

Since Fama and French (1988), the predictability of long-term returns has been a much discussed stylized fact in empirical finance. For exogenous predictors, Stambaugh (1999) and Campbell (2001) point out the effect of the persistence of the predictor on the evidence of long-run predictability. BP show that long-term variance, measured by realized variance, can predict long-term returns. We extend their analysis, allowing for infrequent jumps in returns. 
Given estimates for $C_{t-h, t}$ and $\mathcal{J}_{t-h, t}$, we can now estimate the coefficients in Eqn. (1) which links h-month excess returns to the previous h-month continuous volatility and jumps. Table 3 reports these point estimates, and below, the standard HAC corrected $t$-statistic, and the rescaled $t$-statistic, $t / \sqrt{T}$. Valkanov (2003) shows that the standard $t$-statistic overstates the significance of long-horizon relationships, and is not asymptotically normally distributed under the null hypothesis of no predictability. Therefore, conventional critical values lead to incorrect inference. The rescaled $t$-statistics remedies this problem. We show in the next subsection how to simulate critical values for the rescaled $t$-statistics. We first discuss the empirical evidence.

The bottom panel of Table 3 shows the results with an intercept in the regression. As per the rescaled t-statistic, the impact of the jump on future excess returns, $\gamma_{h}$, is insignificant at all horizons. Curiously enough, the impact of continuous volatility, $b_{h}$, is equally insignificant and sometimes even negative, apart from at the very long horizons which are probably not reliable. ${ }^{3}$

In long-run regressions, with a horizon sizable relative to the sample period, the issue of efficiency is crucial. Our regressions may simply lack precision. In their analysis of the predictability of stock returns, Campbell and Thompson (2008) show that weak restrictions on the coefficients, consistent with economic common sense, such as the need for a positive risk premium, generally lead to better forecasts. In our case, a zero-intercept in Eqn. (1) should yield slope coefficient estimates with increased precision. The zero-intercept restriction is consistent with Merton's (1980) proportional link between the conditional mean and variance of stock market returns.

The top panel in Table 3 displays the results of the no-intercept regressions. If this restriction is reasonable, the slope coefficients are now estimated with increased precision. Indeed, the estimates of the continuous volatility coefficients $b_{h}$, are now positive at all horizons and both the standard and rescaled t-statistics point at statistical significance for horizons above 3 months. Note also how $\widehat{b}_{h}$ appears stable across horizon, remaining between 1.4 and 2.6 for all horizons including the very long ones. This is in contrast with the bottom panel, regressions with intercept, showing large swing in point estimates and large estimates for horizons above 6 years, all unreliable.

The jump coefficients $\widehat{\gamma}_{h}$ are however still insignificant across horizons. Yet they are larger

\footnotetext{
${ }^{3}$ We report these ultra-long horizons, e.g., above 8 years, for comparability with the existing literature.
} 
than the continuous volatility estimates $\widehat{b}_{h}$, for example 8 versus 2 at a 6 -year horizon. One needs to keep in mind the economic magnitude of these point estimates. A 5-year continuous volatility higher by $1 \%$, implies a 5 -year excess return higher by $2.1 \%$. A 5 -year jump higher by $1 \%$ implies a 5 -year excess return higher by $5.4 \%$. However, as Table 1 shows, the average monthly jump size is less than a tenth of the continuous volatility. Further, jumps are far less persistent than continuous volatility implying even smaller average multi-period jump sizes.

To summarize, the contribution of past jumps to the predictable part of medium- to longterm excess returns is marginal relative to that of continuous volatility. These results seem to suggest that realized jumps are not a state variable driving the risk premium dynamics. The continuous component of the total quadratic variation appears to be the major determinant of the risk-return trade-offs.

\subsection{Critical Values of the Rescaled t-Statistic}

We now explain how we compute the critical values of the rescaled t-statistic in Table 3. Long-run regressions with overlapping errors are notorious for potentially overstating predictability. Valkanov (2003) writes that " long-horizon regressions will always reveal significant results, whether or not there is a structural link between the underlying variables". He argues that the rolling summation of a stationary series creates a long-horizon variable behaving asymptotically as a series with a stochastic trend. Consequently, standard test procedures for the detection of long-run relationships are inaccurate, and new test statistics are required. An analytical inspection of the asymptotic distribution of the usual Student-t, through the Functional Central Limit Theorem, shows that

it does not converge to a well-defined distribution. However, the rescaled t-statistic, that is, the Student-t divided by the square root of the sample size, can be shown to be adequate for testing long-horizon regressions. Furthermore, the simplicity of simulating the limiting distribution of the rescaled t-statistic's compensates for its asymptotic non normality.

While the continuous component of realized variance is serially correlated, the jump component is distinctly less persistent, see Figure 4. These different dynamics may cause the rescaled t-statistics for the slope estimators in the regression of long-term returns $R_{t, t+h}$ on past continuous 
volatility $C_{t-h, t}$ and jumps $\mathcal{J}_{t-h, t}^{2}$ to behave differently

A diagnostic of the statistical significance of slope coefficients based on rescaled t-statistics, requires the simulation of critical values. To do this, we jointly simulate monthly excess returns, continuous volatility and jumps under the assumption of no predictability. Then, we aggregate these simulated monthly data and run the multi-period regression as on the market data. Specifically, we simulate monthly variables as

$$
\left(\begin{array}{c}
R_{t+1} \\
C_{t+1} \\
\mathcal{J}_{t+1}^{2}
\end{array}\right)=\left(\begin{array}{c}
0 \\
\alpha_{C} \\
\alpha_{J}
\end{array}\right)+\left(\begin{array}{ccc}
0 & b_{1} & \gamma_{1} \\
0 & \rho_{11} & \rho_{12} \\
0 & \rho_{21} & \rho_{22}
\end{array}\right)\left(\begin{array}{c}
R_{t} \\
C_{t} \\
\mathcal{J}_{t}^{2}
\end{array}\right)+\left(\begin{array}{c}
e_{t+1} \\
u_{t+1}^{C} \\
u_{t+1}^{\mathcal{J}}
\end{array}\right)
$$

Under the null hypothesis of $H_{0}$ no predictability, $b_{1}=0, \gamma_{1}=0$, and the errors $e_{t+1}, u_{t+1}^{C}, u_{t+1}^{\mathcal{J}}$ have no autocorrelation. They can however be contemporaneously correlated. We denote their standard deviations $\left(\sigma_{e}, \sigma_{u^{C}}, \sigma_{u^{J}}\right)$ and their covariances, $\left(\sigma_{e u^{C}}, \sigma_{e u^{J}}, \sigma_{u^{C} u^{J}}\right)$.

This extends BP's (2008) parametric bootstrap. The use of a Normal VAR(1) to model the joint dynamics of returns and the two components of realized variance is parsimonious in this context. The VAR(1) model is not restrictive since a higher-order VAR system can be re-expressed as a first order, see Campbell and Shiller (1988). However, we are aware that the normal errors may be more suitable to the modeling of the logarithm of realized volatility and its components. Our trivariate $\operatorname{VAR}(1)$ model is also related to the one in Busch, Christensen and Nielsen (2011), who model the incremental information of implied volatility relative to both the continuous and jump component of realized volatility for different markets. The analogy lies in specifying a VAR model for the split components of realized volatility. However, there is a major difference: Busch et al. (2011) investigate the link with implied volatility, they do not consider risk vs. return models.

We can now generalize BP's local-to-unity framework to the case of two regressors with different persistence. We assume the portion of overlap $h=\lfloor\lambda T\rfloor$ to be a non-trivial constant fraction of the sample size where $\lfloor$.$\rfloor denotes the lesser greatest integer operator. Then, we set$ $\rho_{i i}=1+c_{i} / T$ and $\rho_{12}=\rho_{21}=0$. The parameters $c_{i}(i=1,2)$ controls the deviations from the 
unit root. Propositions 1 and 2 in Appendix A give the theoretical foundation to construct the adequate test statistics and analizing their asymptotic properties.

We generate 10,000 paths of 696 months (58 years). In all the simulations, we have $\alpha_{C}=$ $\alpha_{J}=0, \rho_{12}=\rho_{21}=\rho_{22}=0$. We set $\sigma_{u^{C}}=\sigma_{u^{J}}=1, \rho_{e u^{J}}=-0.25$ and $\rho_{u^{C} u^{J}}=0.7$. We conduct two sets of simulations. In the first set, $\mathrm{S}_{1}$, the autocorrelation of the continuous volatility $\rho_{11}$, is 0.75 , and the correlation between excess returns and continuous volatility shocks $\rho_{e u^{C}}$, is -0.3 , consistent with the data. In the second set, $\mathrm{S}_{2}$, we have $\rho_{11}=0.99$ and $\rho_{e u} C=-0.7$. The second set induces more persistence in volatility and a higher (negative) value of $\rho_{e u^{C}}$ consistent with some empirical findings, such as Jacquier, Polson and Rossi (2004). The negative correlation is a well known feature of index volatility, sometimes denoted the leverage effect, a misnomer. Both sets impart a small amount of negative correlation between jump and return shocks and a positive correlation between jump and continuous volatility shocks.

Recall that the model in Eqn. (1) is estimated for horizons from 1 to 120 months. We consider zero-intercept regressions, potentially more precise. We collect the $5 \%$ critical values for both $b_{h}=0$ and $\gamma_{h}=0$. Table 4 shows the critical values for $t_{\widehat{b}_{h}} / \sqrt{T}$ and $t_{\widehat{\gamma}_{h}} / \sqrt{T}$. There is very little difference between the two simulation sets $S_{1}$ and $S_{2}$. We use the values from set $S_{1}$ to flag the statistical significance of the rescaled t-statistic in Table 3.

For the unconstrained regressions, $t_{\widehat{b}_{h}} / \sqrt{T}$ values suggest that the link between excess return and past continuous volatility is insignificant at 5\% level for all but the two extreme horizons 108 and 120 months. While we include these horizons for comparability with other studies, any evidence at a 10 year horizon based on a 58 year sample should be looked upon with a healthy degree of skepticism. When the intercept is constrained at 0 , the rescaled t-statistics for the continuous volatility in Table 3 are now outside their confidence bounds at all horizons beyond 3 months. However, there is no such evidence for jump risk: all the $t_{\widehat{\gamma}_{h}} / \sqrt{T}$ values lie inside their confidence intervals. We fail to detect any significant impact of jumps on future market excess returns.

We now turn to joint restriction tests on the coefficients, using a Wald test statistic based upon the joint asymptotic distribution of the slope coefficients for all $h \mathrm{~s}$. 


\section{Horizon Effects in the Risk vs Return Relationship}

Continuous volatility is the component of the realized volatility which explains a significant part of medium- to long-term market excess returns. This suggests that diffusion risk is systematic; investors require a premium for bearing this risk. We now focus on the estimation of the trade-off intensity between the market excess return and the continuous volatility across horizons. The set of slope coefficients estimated at various horizons is labeled as the "term structure of risk-return tradeoffs". There is a horizon effect in the term structure when the trade-off intensities are significantly different across horizons.

It is possible to relate our empirical characterization of the term structure of trade-offs to Merton's (1973) ICAPM implications. The absence of a horizon effect in the term structure of riskreturn trade-offs is consistent with an ICAPM with a constant proportionality between conditional first and second moments of excess returns on the market portfolio. We now investigate the presence of a horizon effect in the term structure of risk-return trade-offs.

\subsection{Empirical Model}

In Merton's (1973) ICAPM, expected returns of individual assets depend on their covariances with the market portfolio and with state variables that span the investment opportunity set. In the aggregate, see Merton (1980), the conditional expected excess return of the market portfolio is proportional to its conditional variance, namely $E_{t}\left[R_{t+1}\right]=A \times \operatorname{Var}_{t}\left[R_{t+1}\right]$. The proportionality constant $A$ is the relative risk aversion of a representative investor with iso-elastic preferences.

The results of Section 3 show that jumps have a very marginal impact on medium- to longterm excess returns. Hereafter, we use continuous volatility as the sole predictor of long-run excess

returns. To assess whether the relation between future market excess returns and past continuous volatility varies with the investment horizon, we estimate the regressions

$$
R_{t, t+h}=\alpha_{h}+\beta_{h} C_{t-h, t}+\eta_{t, t+h}
$$


where $R_{t, t+h}$ is the excess return, $C_{t-h, t}$ is the past market continuous volatility, and $\eta_{t, t+h}$ is the overlapping prediction error. In Eqn. (12), we use the past market continuous volatility as the predictor instead of the lagged realized variance as in BP (2008). For the same reason as in section 3.1, we perform a regression with no-intercept as well as one with intercept. We already noted the beneficial effect of this constraint in Table 3 . The coefficient $\beta_{h}$ is a $h$-horizon proxy for a representative investor's relative risk aversion. We can interpret the coefficients $\beta_{h}$ as a term structure of market prices of diffusion risk, see Campbell and Viceira (2005) for a discussion of the notion of term structure of risk-return trade-offs. Table 5 displays estimation results for Eqn. (12).

With respect to continuous volatility, the findings in Table 5 are similar to those in Table 3. Again, the presence of an intercept renders the estimation quite noisy. In contrast, with the zero intercept constraint, the slope coefficients are positive and statistically significant at all horizons except for monthly and quarterly. Above the quarterly horizon, the slope coefficients take values in a relatively tight range from 1.5 to 3.2 , pointing to a possibly flat term structure. In the context of Merton's (1973) ICAPM, the slope coefficients in Table 5 point are consistent with reasonable, perhaps even low, values for the relative risk aversion coefficient.

One may be puzzled by the absence of significant relationship at short (monthly and quarterly) horizons. This may come from high levels of volatility during some periods of financial turmoil. For instance, monthly market variances in October 1987, the fourth quarter of 2008 and the first quarter of 2009 were extreme, 40 to 50 times larger than the average variance for the entire post-war period. Therefore, the empirical evidence of risk-return trade-off may be more sensitive to extreme volatilities, at shorter than longer horizons. The 58 years in our empirical analysis contain two major episodes of extreme volatility in the short run. This could make the assessment of the risk-return trade-off very imprecise. To verify if this is the reason for our puzzling slope estimates up to quarterly horizons, we repeat the regressions, now using a $99 \%$ winsorized continuous volatility. The bottom panel of Table 5 shows the results. Indeed, the coefficients at the monthly and quarterly horizons now have magnitudes similar to the longer horizons estimates. 


\subsection{Test of Equality of the Slope Coefficients Across Horizons}

In this subsection, we present inference and simulation results for restriction tests on the term structure of risk-return trade-offs. Campbell and Viceira (2005) show how to extract this term structure from a parsimonious model of return dynamics. They use 50 years of post-war quarterly data from the U.S. stock and bond markets to illustrate their approach. In our local-to-unity framework, we rely on a normalized version of the classical Wald test statistic (of equality) to conduct inference. This rescaled Wald statistic exhibits proper convergence properties and is well-behaved in finite sample.

\subsubsection{Inference}

We consider a restricted version of the system in Eqn. (11), eliminating the jump variable. Specifically, consider

$$
R_{t+1}=\beta_{1} C_{t}+\epsilon_{t+1}
$$

and

$$
C_{t+1}=\rho_{0}+\rho_{1} C_{t}+u_{t+1}
$$

with $\rho_{0}=0$ and $\rho_{1}=1+c / T$. Deviations from the unit root are controlled by the parameter $c$ at the decreasing rate $T$ and the condition $\lim _{T \rightarrow \infty} h / T=\lambda$ is satisfied. The errors $\epsilon_{t+1}$ and $u_{t+1}$ are as defined for Eqn. (11), with variances and covariances $\sigma_{\epsilon}^{2}, \sigma_{\epsilon u}$ and $\sigma_{u}^{2}$.

When the return and the continuous volatility follow the dynamics in Eqns. (13-14), under the null hypothesis of no predictability $\left(\beta_{1}=0\right)$, we can derive the asymptotic distribution of the

term structure of risk-return trade-offs $\underline{\widehat{\beta}}=\left(\widehat{\beta}_{h_{1}}, \ldots, \widehat{\beta}_{h_{K}}\right)$. We can also compute the limiting approximation of its covariance matrix $\operatorname{Var}(\underline{\hat{\beta}})$. It is important to understand that one needs to compute the asymptotics in order to identify the proper convergence rate for the test statistics. We refer the reader to Propositions 3 and 4 in the APPENDIX A for technical details. 
For a given restriction $\mathcal{R}$, the rescaled wald test statistic is computed as

$$
T^{-1} \chi_{(\mathcal{R})}^{2}=T^{-1}\left\{(\mathcal{R} \underline{\hat{\beta}})^{\prime}\left[\mathcal{R} \operatorname{Var}(\underline{\hat{\beta}}) \mathcal{R}^{\prime}\right]^{-1}(\mathcal{R} \underline{\hat{\beta}})\right\}
$$

Before looking at the horizon effect, we consider the joint test of no predictability $H_{0}$ : $\left\{\beta_{h_{i}}=0\right.$ for $\left.i=1, \ldots, K\right\}$ against $H_{1}: \beta_{h_{j}} \neq 0$ for some horizon $h_{j}$. Though it is common to assess predictability in returns using separate rescaled t-statistics, an improvement of this procedure is to rely on the rescaled Wald test statistic. The rescaled Wald statistic allows for a simultaneous inference. Thus, it helps circumvent the challenge of controlling for the test size in multiple hypotheses testing. In this case, the $K \times K$ identity matrix $I_{K}$ defines the set of restrictions. The calculated rescaled Wald statistic $T^{-1} \chi_{\left(I_{K}\right)}^{2}=66.7$ for the regressions in Eqn. (12), and is greater than its simulated $5 \%$ right-tail critical value of 2.242 . Similarly, $T^{-1} \chi_{\left(I_{K}\right)}^{2}=5.49$ is larger than its critical value of 1.39 for the zero-intercept regressions in Eqn. (12). These findings support evidence against the null hypothesis of no predictability in the risk-return relationship.

One can compare slope coefficients across horizons by assessing the degree of statistical discrepancy between the estimates. A flat term structure of risk-return trade-offs, which illustrates the absence of a horizon effect, should imply statistically insignificant differences between slope point estimates at various horizons. As a first attempt to the horizon effect detection, we look at pairwise comparisons of slope coefficients from the term structure of risk-return trade-offs in Eqn. (12). The rescaled Wald statistic for testing the difference between two trade-off slope coefficients ( $H_{0}: \beta_{h_{i}}=\beta_{h_{j}}$ against the alternative $H_{1}: \beta_{h_{i}} \neq \beta_{h_{j}}$ for $i \neq j$ ) is computed as a standard restriction test statistic. Specifically, for the unrestricted regressions, we have:

$$
T^{-1} \chi_{\left(\widehat{\beta}_{h_{j}=\left\lfloor\lambda_{j} T\right\rfloor}^{2}-\widehat{\beta}_{h_{i}=\left\lfloor\lambda_{i} T\right\rfloor}\right)} \stackrel{\mathcal{L}}{\longrightarrow} \frac{\bar{N}}{\bar{D}} .
$$

For the restricted regressions $\alpha_{h}=0$, we have:

$$
T^{-1} \chi_{\left(\widehat{\beta}_{h_{j}=\left\lfloor\lambda_{j} T\right\rfloor}^{2}-\widehat{\beta}_{h_{i}=\left\lfloor\lambda_{i} T\right\rfloor}\right)} \stackrel{\mathcal{L}}{\longrightarrow} \frac{N}{D} .
$$


As the sample size grows to infinity $(T \rightarrow \infty)$, the rescaled Wald statistic for testing the difference between slopes estimated at two distinct horizons has a non-degenerate distribution. We propose a precise definition of the limiting distributions $\frac{\bar{N}}{\bar{D}}$ and $\frac{N}{D}$ in the APPENDIX B.

Alternatively, a joint restriction test offers a unified answer when examining the horizon effect in the term structure of risk-return trade-offs. In the case of a flat term structure, we should not find enough evidence to reject the null hypothesis of trade-off intensities being equal across horizons $\left(H_{0}: \beta_{h_{1}}=\beta_{h_{2}}=\cdots=\beta_{h_{K}}\right)$. Under this assumption, the rescaled Wald statistic $T^{-1} \chi_{(\mathcal{R})}^{2}$ characterizes $K-1$ restrictions $\left\{\beta_{h_{i}}-\beta_{h_{1}}=0\right.$ for $\left.i=2, \ldots, K\right\}$.

\subsubsection{Empirical Results And Simulated Critical Values}

We now report the results of these tests. Significance is obtained for test statistics that are greater than their simulated critical values. Panel (a) in Table 6 presents rescaled Wald statistics of the pairwise differences of slope coefficients int the NYSE/AMEX long-run regressions of Eqn. (12) for horizons $h$ from 1 to 120 months. Panel b shows the relevant right tail $5 \%$ critical values obtained by simulation. We also use a Bonferroni correction to control for testing multiple dependent hypotheses, as for example in Campbell and Yogo (2006). The simulated data mimic the empirical features of the NYSE/AMEX data under the null hypothesis. Notable variations in the slope values from horizon $h_{i}$ to horizon $h_{j}$, should result in larger $T^{-1} \chi_{\left(\widehat{\beta}_{h_{j}}-\widehat{\beta}_{h_{i}}\right)}^{2}$ possibly lying outside its critical value.

For the unrestricted regressions, the empirical results in Panel (a) show insignificant differences between risk-return trade-off slopes up to 7 years. The rescaled Wald test statistics are all within their simulated confidence bands for $h_{i}$ below 72 months and $h_{j}$ below 84 months. The only significant rescaled Wald statistics are for $h_{i}$ less than 84 and $h_{j}=96,108,120$. These results are in line with the evidence in Table 3 of much larger coefficients for the very long horizons above 84 months. For both very high $h_{i}$ and $h_{j}$, the slope estimates are significant but close to each other. Thus, the bottom right area in Panel (a) of Table 5 presents insignificant $T^{-1} \chi_{\left(\widehat{\beta}_{h_{j}}-\widehat{\beta}_{h_{i}}\right)}^{2}$ test statistics for $h_{i}=96,108,120$ and $h_{j}=108,120$. 
Consider now the zero-intercept regressions that can deliver slope estimates with increased precision. Interestingly, for the zero-intercept regressions, we notice significant, positive and similar slope coefficients beyond the quarterly horizon. Panel (a) of Table 7 shows the estimates. Consequently, we only find rejection of equality by our test statistic $T^{-1} \chi_{\left(\widehat{\beta}_{h_{j}}-\widehat{\beta}_{h_{i}}\right)}$ for $h_{i}=1,3$ and $h_{j}=6, \ldots, 120$. This is consistent with a flat risk-return term structure beyond 3 months. The significant difference between under versus over 3 months slope estimates could lead to the conclusion that there is a horizon effect in the term structure of risk return trade-offs. However, recall our sensitivity analysis in section 4.1, where we found the slope estimates below a quarter to be sensitive to extreme short-term volatility values in the sample.

We verify this again by using the $99 \%$ winsorized continuous volatility in the zero-intercept regression in (12). The weak short term risk-return slope coefficients are now larger, in fact positive and statistically significant. The second and third panels in Table 5 show this remarkable increase in monthly (quarterly) slope point estimate from -0.54 ( quarterly 0.49 ) to 1.97 (quarterly 2.49). The regressions at horizons longer than 3 months are more robust to extreme values in volatility, as shown by the similar slope estimates in the middle and bottom panels of Table 5. Panel (b) of Table 7 shows the pairwise comparison tests based upon slope estimates from the winsorized continuous volatility. We now fail to detect statistically significant differences between pairs of coefficients at any horizons including monthly and quarterly. Table 8 contains the simulated critical values for pairwise equality test statistic based upon zero-intercept regressions. The corresponding rescaled Wald statistic $T^{-1} \chi_{(\mathcal{R})}^{2}$ for the joint restriction test, confirms this conclusion. Its calculated value (0.068) is far less than its simulated $5 \%$ critical value $(0.737)$. There is no evidence against a flat term structure of risk-return trade-offs for the set of horizons considered in our analysis.

To summarize, the zero-intercept regressions are consistent with a positive and proportional relationship between market excess return and past market continuous realized variance, constant across horizons. Assuming that these variables are reasonable substitutes for the conditional expected excess return and continuous volatility of the stock market, these findings are consistent with Merton's (1973) ICAPM implications. When jumps are filtered out from the total quadratic variation, we get a flat term structure of risk-return trade-offs. From our empirical results, there is 
no clear evidence that a representative investor with power utility preference modifies his relative risk aversion as he undertakes longer term investments.

\section{Conclusion}

In this paper, we segregate past market variance into a continuous and a jump components. We use the asymptotics of realized volatility to compute realized variance, realized continuous volatility, and jump estimates. Our high frequency for the purpose of these computations is daily returns. We compute these variables for multiple horizons from one to 120 months. We then estimate the link between future market excess returns and past continuous variance and jumps at these horizons. We provide the asymptotic analysis for the use of the rescaled t-statistic which is better behaved than the standard heteroskedasticity and autocorrelation consistent (HAC) correction of the ordinary t-statistic. We also simulate its exact sample distribution as the long horizons constitute a sizable fraction of the sample size.

Relying on this inference, we find that the past jump component does not contribute the time variation of future excess return at most horizons. The entirety of the previously documented predictive power of realized variance on future excess returns is due to the continuous volatility. Using continuous volatility, risk-return trade-offs are significant at medium to long horizons. These findings point to continuous volatility as a central determinant of long-run risk-return relationships.

We then inspect the term structure of risk-return trade-offs after extracting jumps from the market variances, that is, using only continuous realized variance as the predictor. We develop a rescaled Wald statistic to test the presence of a horizon effect possibly induced by different risk aversions for a representative investor facing different investment horizons. Our results are consistent with a flat term structure of risk-return trade-offs, that is, the same risk aversion for different horizons of a Merton style ICAPM.

We use regressions with and without intercept, where the zero-intercept constraint is consistent with a return vs. risk model as in Merton (1980). The estimates arising from the unconstrained regressions are unstable. This is a case where, as in Campbell and Thompson (2008), imposing a 
reasonable model-based constraint is beneficial. Further, even with a zero intercept, we initially find a puzzling lack of connection between variance and returns at the shorter horizons. When we repeat the estimation with a winsorized continuous volatility to attenuate some episodes of extreme short-term volatility, the resulting short-horizon slope coefficients are in line with the longer horizons. Indeed, at small horizons the empirical risk vs return relationship is unstable due to high variability. Removing these sources of instability improves the estimation.

An interesting area of future research would be to study the out-of sample version of these results, possibly using asset allocation to calibrate their economic significance. Also, it may be interesting to explore the robustness of our findings on the (lack of) impact of past jumps on future returns to the jump specification used. Our data analysis reveals a relatively small number of months with jumps, and an interesting lack of connection between the size of realized variance and the probability of occurrence of a jump (Figure 1 bottom panel). Our extraction technique is adapted to finite activity jumps, and within this class we explored robustness with the significance level of the $\mathrm{z}$ statistic. The link between risk and return might be interpreted differently if one entertained for example infinite activity pure jump levy processes such as in Carr et al. (2002). 


\section{REFERENCES}

Aït-Sahalia, Y. (2004). "Disentangling Diffusion from Jumps." Journal of Financial Economics 74, 487-528.

Aït-Sahalia, Y., and Jacod, J. (2009). "Testing For Jumps In a Discretely Observed Process." Annals of Statistics 37, 184-222.

Andersen, T. G., Bollerslev, T., and Diebold, F. X. (2007), "Roughing It Up: Including Jump Components in the Measurement, Modeling and Forecasting of Return Volatility." Review of Economics and Statistics 89, 701-20.

Andersen, T. G., Bollerslev, T., and Diebold, F. X. (2010). "Parametric and Nonparametric Volatility Measurement." in Y. Ait-Sahalia (eds.) and L.P. Hansen, Handbook of Financial Econometrics. Vol. 1, Elsevier Science, Amsterdam, The Netherlands, pp. 67-138.

Andersen, T. G., Bollerslev, T., Diebold, F. X., and Labys, P. (2003). "Modeling and Forecasting Realized Volatility." Econometrica 71, 579-625.

Andersen, T. G., Bollerslev, T., and Meddahi, N. (2004). "Analytical Evaluation of Volatility Forecasts." International Economic Review 45, 1079-1110.

Andersen, T. G., Dobrev, D. P., and Schaumburg, E. (2012). "Jump-Robust Volatility Estimation using Nearest Neighbor Truncation." Journal of Econometrics 169, 75-93.

Back, K. (1991). "Asset Pricing For General Processes." Journal of Mathematical Economics 20, 371-395.

Bali, T. G., and Engle, R. F. (2010). "The Intertemporal Capital Asset Pricing Model with Dynamic Conditional Correlations." Journal of Monetary Economics 57, 377-390.

Ball, C. A., and Torous, W. N. (1983). "A Simplified Jump Process for Common Stock Returns." Journal of Financial and Quantitative Analysis 18, 53-65.

Bandi, F. M., and Perron, B. (2008). "Long-Run Risk-Returns Trade-Offs." Journal of Econometrics 143, 349-374.

Bansal, R., and Yaron, A. (2004). "Risks for the Long-Run: a Potential Resolution of Asset Pricing Puzzles." Journal of Finance 59, 1481-1509.

Barndorff-Nielsen, O. E., and Shephard, N.(2004). "Power and Bipower Variation with Stochastic Volatility and Jumps (with discussion)." Journal of Financial Econometrics 2, 1-48.

— (2006). "Econometrics of Testing for Jumps in Financial Economics using Bipower Variation." Journal of Financial Econometrics 4, 1-30.

Bates, D. S. (2000). "Post-'87 Crash fears in the S\&P 500 Futures Option Market." Journal of Econometrics 94, 181-238.

Bollerslev, T., Sizova, N., and Tauchen, G. (2012). "Volatility in Equilibrium: Asymmetries and Dynamic Dependencies." Review of Finance 16, 31-80.

Bollerslev, T., Tauchen, G., and Zhou, H. (2009). "Expected Stock Returns and Variance Risk Premia." Review of Financial Studies 22, 4463-4492.

Bollerslev, T., and Todorov, V. (2010). "Jumps and Betas: A New Framework for Disentangling and Estimating Systematic Risks." Journal of Econometrics 157, 220-235.

Boudoukh, J., Richardson, M., and Whitelaw, R.F. (2008). "The Myth of Long-Horizon 
Predictability." Review of Financial Studies 21, 1577-1605.

Busch, T., Christensen, B. J., and Nielsen, M. Ø. (2011). "The Role of Implied Volatility in Forecasting Future Realized Volatility and Jumps in Foreign Exchange, Stock, and Bond Markets." Journal of Econometrics 160, 48-57.

Campbell, J.Y. (2001). "Why Long Horizons? A Study of Power Against Persistent Alternatives." Journal of Empirical Finance 8, 459-491.

Campbell, J. Y., Thompson, S. B. (2008). "Predicting the Equity Premium out of Sample: Can Anything Beat the Historical Average." Review of Financial Studies 21, 1509-1531.

Campbell, J. Y., and Shiller, R. (1988). "The Dividend-Price Ratio and Expectations of Future Dividends and Discount Factors" Review of Financial Studies 1, 195-227.

Campbell, J. Y., and Viceira, L. (2005). "The Term Structure of the Risk-Return Trade-off." Financial Analysts Journal 61, 34-44.

Campbell, J. Y., and Yogo, M. (2006). "Efficient Tests of Stock Return Predictability." Journal of Financial Economics 81, 27-60.

Carr, P., Geman, H., Madan, D., and Yor, M. (2002) "The Fine Structure of Asset Returns: An Empirical Investigation", Journal of Business 75(2), 305-332

Carr, P., and Wu, L. (2003a). "The Finite Moment Log Stable Process And Option Pricing." Journal of Finance 58, 753-777.

Carr, P., and Wu, L. (2003b). "What Type of Process Underlies Options? A Simple Robust Test." Journal of Finance 58, 2581-2610.

Corsi, F. (2009). "A Simple Approximate Long-Memory model of Realized Volatility" Journal of Financial Econometrics 7, 174-196.

DeGennaro, R. P., and Zhao, Y. L. (1998). "Stock Returns and Volatility: Another Look." Journal of Economics and Finance 22, 5-18.

Eraker, B., Johannes, M., and Polson, N. (2003). "The Impact of Jumps in Volatility." Journal of Finance 58, 1269-1300.

Fama, E. F., and French, K. R. (1988). "Dividend Yields and Expected Stock Returns." Journal of Financial Economics 22, 3-25.

French, K. R., Schwert, W., and Stambaugh, R. F. (1987). "Expected Stock Returns and Volatility." Journal of Financial Economics 19, 3-29.

Ghysels, E., Santa-Clara, P., and Valkanov, R. (2005). "There Is a Risk-Return Trade-Off After All." Journal of Financial Economics 76, 509-548.

Glosten, L.R., Jagannathan, R., and Runkle, D.E. (1993). "On the Relation Between the Expected Value and the Volatility of the Nominal Excess Return on Stocks." Journal of Finance 48, 1779-1801.

Goyal, A. and Welch I. (2008). "A Comprehensive Look at the Empirical Performance of Equity Premium Predictions." Review of Financial Studies 21(4), 1455-1508.

Guo, H., and Whitelaw, R. (2006). "Uncovering the Risk-Return Relation in the Stock Market." Journal of Finance 61, 1433-1464.

Hansen, L. P., and Hodrick, R. J. (1980). "Forward Exchange Rates as Optimal Predictors 
of Future Spot Rates: An Econometric Analysis." Journal of Political Economy 88(5), 829-852.

Huang, X., and Tauchen, G. (2005). "The Relative Contribution of Jumps to Total Price Variance." Journal of Financial Econometrics 3, 456-499.

Jacquier, E., Polson, N. G., and Rossi, P. E. (2004). "Bayesian Analysis of Stochastic Volatility Models with Fat-Tails and Correlated Errors." Journal of Econometrics 122, 185-212.

Jarrow, R. A., and Rosenfeld, E. (1984). "Jump Risk and the Intertemporal Capital Asset Pricing Model." Journal of Business 57, 337-351.

Jiang, G. J., and Oomen, R. C. A. (2008). "Testing For Jumps When Asset Prices Are Observed With Noise-A Swap Variance Approach." Journal of Econometrics 144, 352-370.

Johannes, M. (2004). "The Statistical and Economic Role of Jumps in Continuous-Time Interest Rate Models." Journal of Finance 59, 227-260.

Kim, M. J., Oh, Y. H., and Brooks, R. (1994). "Are Jumps in Stock Returns Diversifiable? Evidence and Implications for Option Pricing." Journal of Financial and Quantitative Analysis 29, 609-631.

Lee, S. S., and Hanning, J. (2010). "Detecting Jumps From Lévy Jump Diffusion Processes." Journal of Financial Economics 96, 271-290.

Lee, S., and Mykland, P. A. (2008). "Jumps in Financial Markets: A New Nonparametric Test and Jump Dynamics." Review of Financial Studies 21, 2535-2563.

Lettau, M., and Ludvigson, S. (2010). "Measuring and Modeling Variation in the RiskReturn Trade-Off." in Handbook of Financial Econometrics, Y. Ait-Sahalia and L.P. Hansen eds., Elsevier Science, Amsterdam, 617-690.

Maheu, J., and McCurdy, T. (2007). "Components of Market Risk and Return." Journal of Financial Econometrics 5, 560-590.

Merton, R.C. (1973). "An Intertemporal Capital Asset Pricing Model." Econometrica 41, 867-887.

Merton, R.C. (1976). "Option Pricing When Underlying Stock Returns Are Discontinuous." Journal of Financial Economics 3, 125-144.

Merton, R.C. (1980). "On Estimating the Expected Return on the Market: An Exploratory Investigation." Journal of Financial Economics 8, 323-361.

Scruggs, J.T. (1998). "Resolving the Puzzling Intertemporal Relation Between the Market Risk Premium and Conditional Market Variance: a Two-Factor Approach." Journal of Finance 53, 575-603.

Stambaugh, R. F. (1999). "Predictive Regressions." Journal of Financial Economics 54, $375-421$.

Tauchen, G., and Zhou, H. (2011). "Realized Jumps on Financial Markets and Predicting Credit Spreads." Journal of Econometrics 160, 102-118.

Valkanov, R. (2003). "Long-horizon Regressions: Theoretical Results and Applications." Journal of Financial Economics 68, 201-232.

Yan, S. (2011). "Jump risk, stock returns and slope of implied volatility smile." Journal of Financial Economics 99 , 216-233. 
Table 1: NYSE/AMEX value weighted index descriptive statistics.

Based on the index monthly returns Jan. 1952 to Dec. 2009. $R V_{t}, C_{t}$ and $\mathcal{J}_{t}^{2}$ are the realized variance, the continuous realized variance and the jumps in monthly returns estimated with a $95 \%$ significance level for the $z$ statistic in Eqn. (9) and (10). $\rho_{1}$ is the first order autocorrelation coefficient. Statistics are not annualized.

\begin{tabular}{lrrrr} 
& $R_{t}$ & $R V_{t}$ & $C_{t}$ & $\mathcal{J}_{t}^{2}$ \\
\hline Mean & 0.0044 & $17 \mathrm{E}-4$ & $16 \mathrm{E}-4$ & $1.4 \mathrm{E}-4$ \\
Variance & 0.0018 & $16.44 \mathrm{E}-6$ & $10.1 \mathrm{E}-6$ & $1.46 \mathrm{E}-6$ \\
Skewness & -0.803 & 11 & 8.4 & 16.7 \\
Kurtosis & 6.154 & 146 & 92 & 298 \\
$\rho_{1}$ & 0.10 & 0.50 & 0.62 & -0.01 \\
$\rho_{1} 1952-1986$ & 0.06 & 0.54 & 0.54 & -0.03 \\
$\rho_{1} 1988-2007$ & -0.01 & 0.50 & 0.50 & -0.02 \\
\hline
\end{tabular}




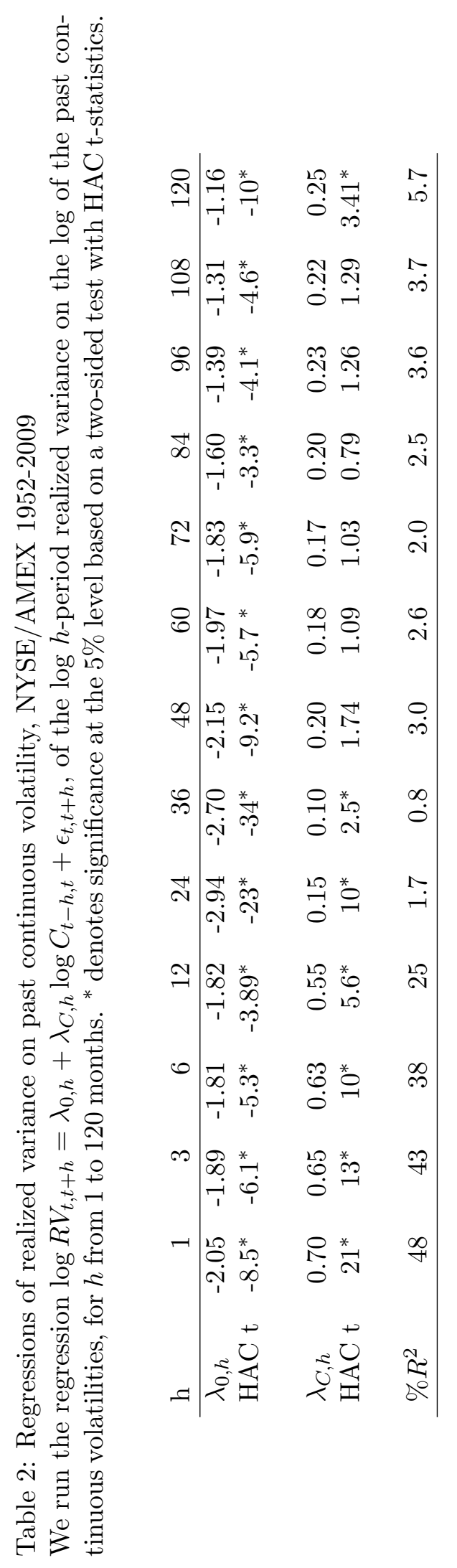




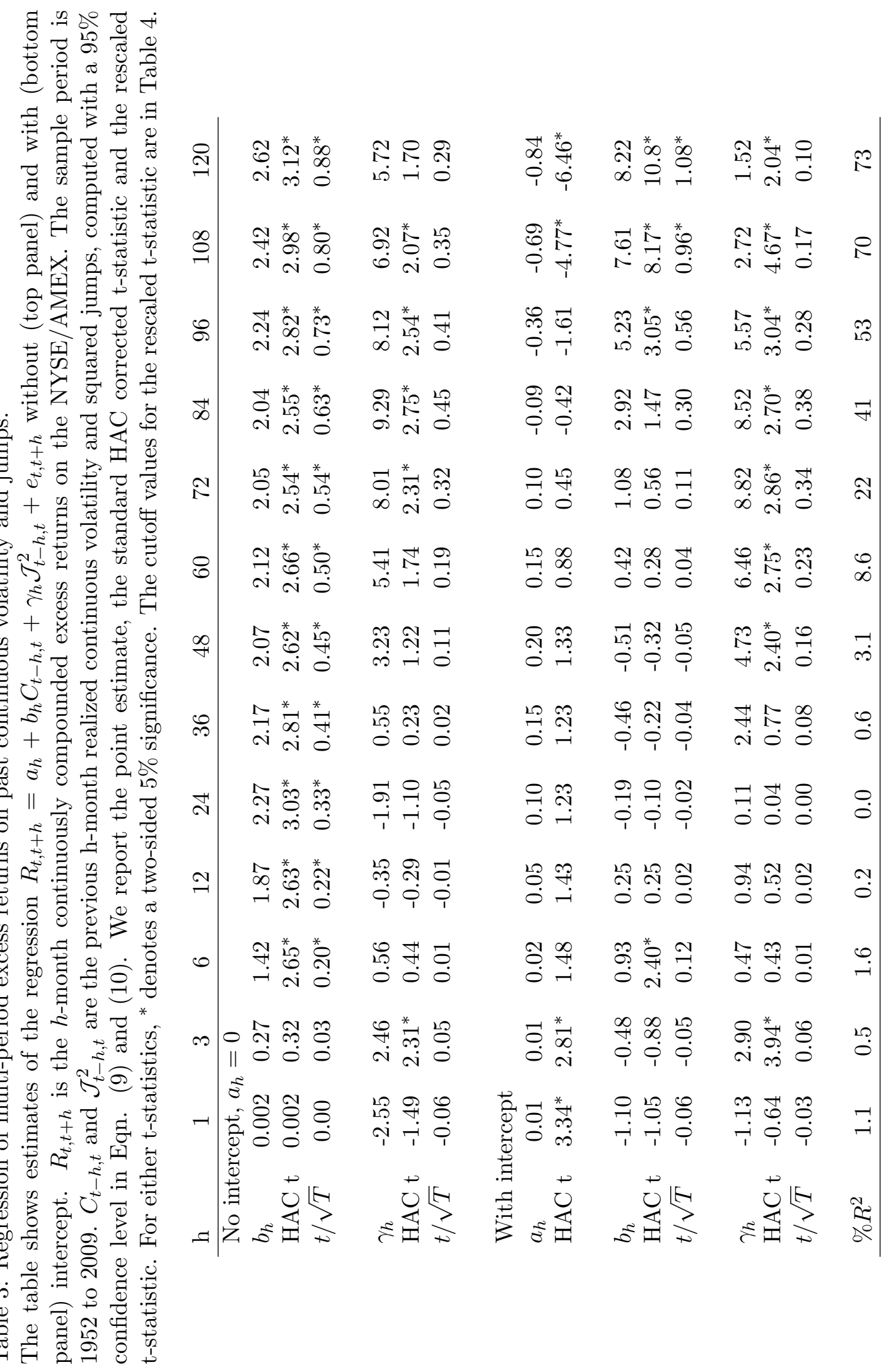




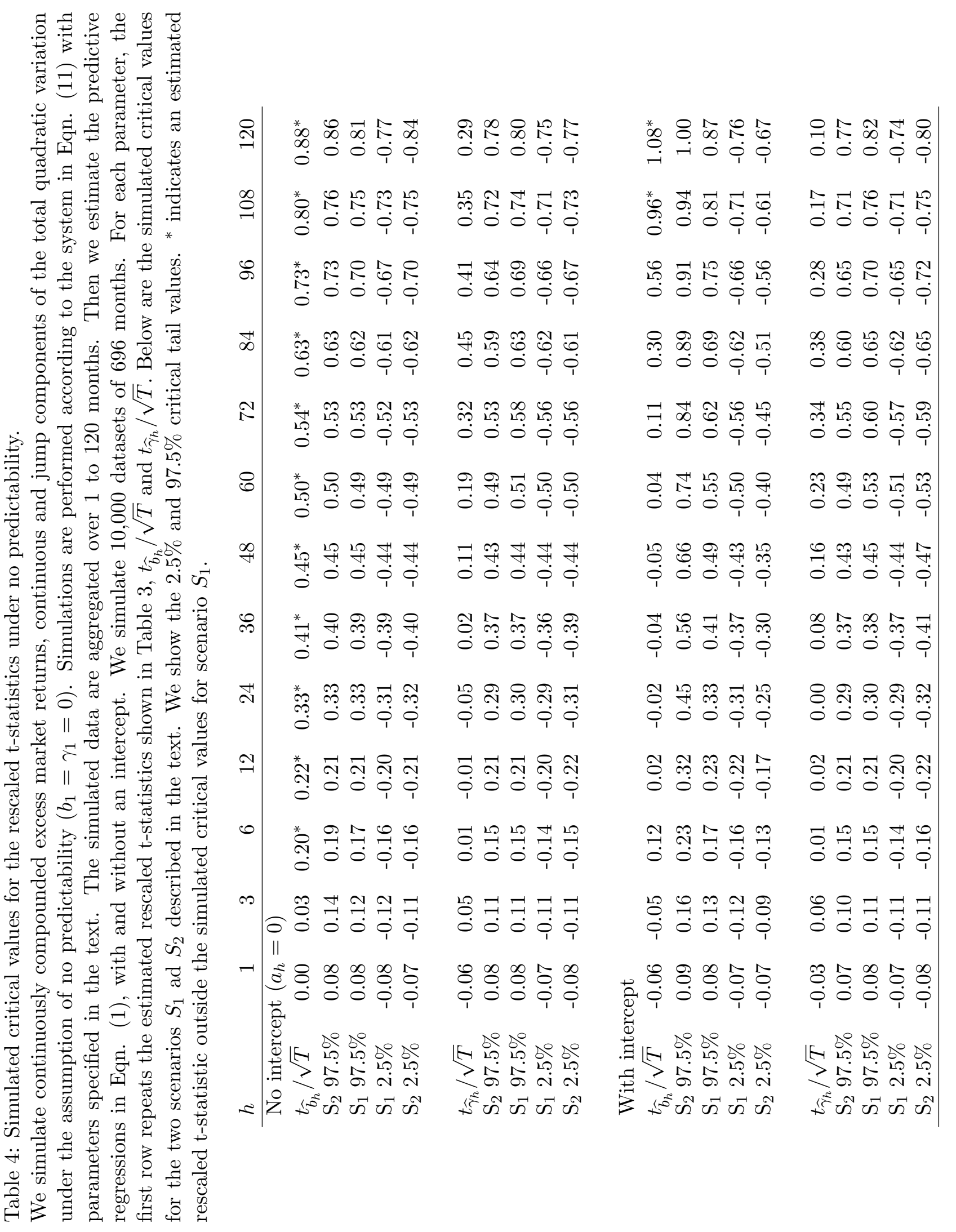




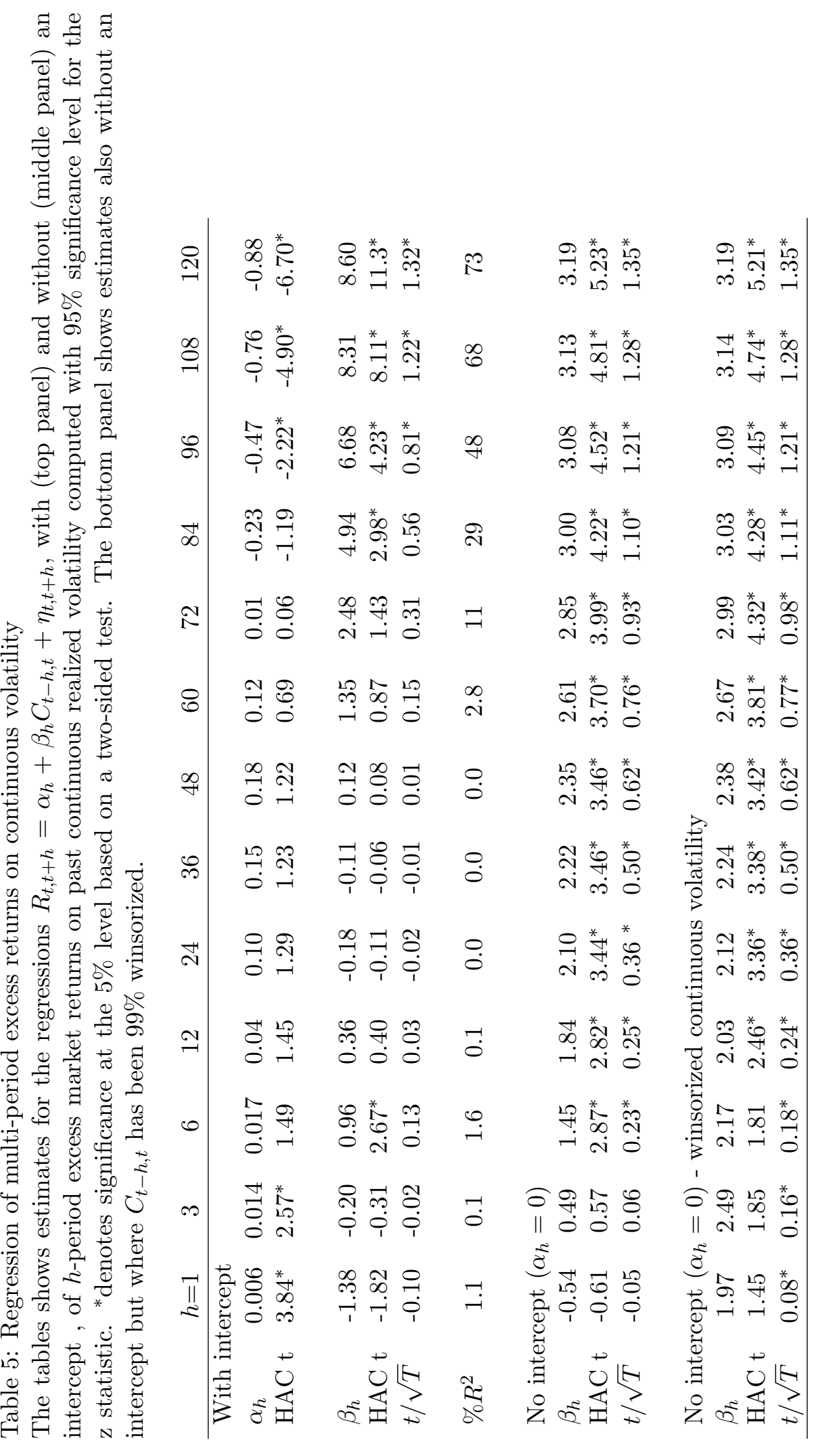




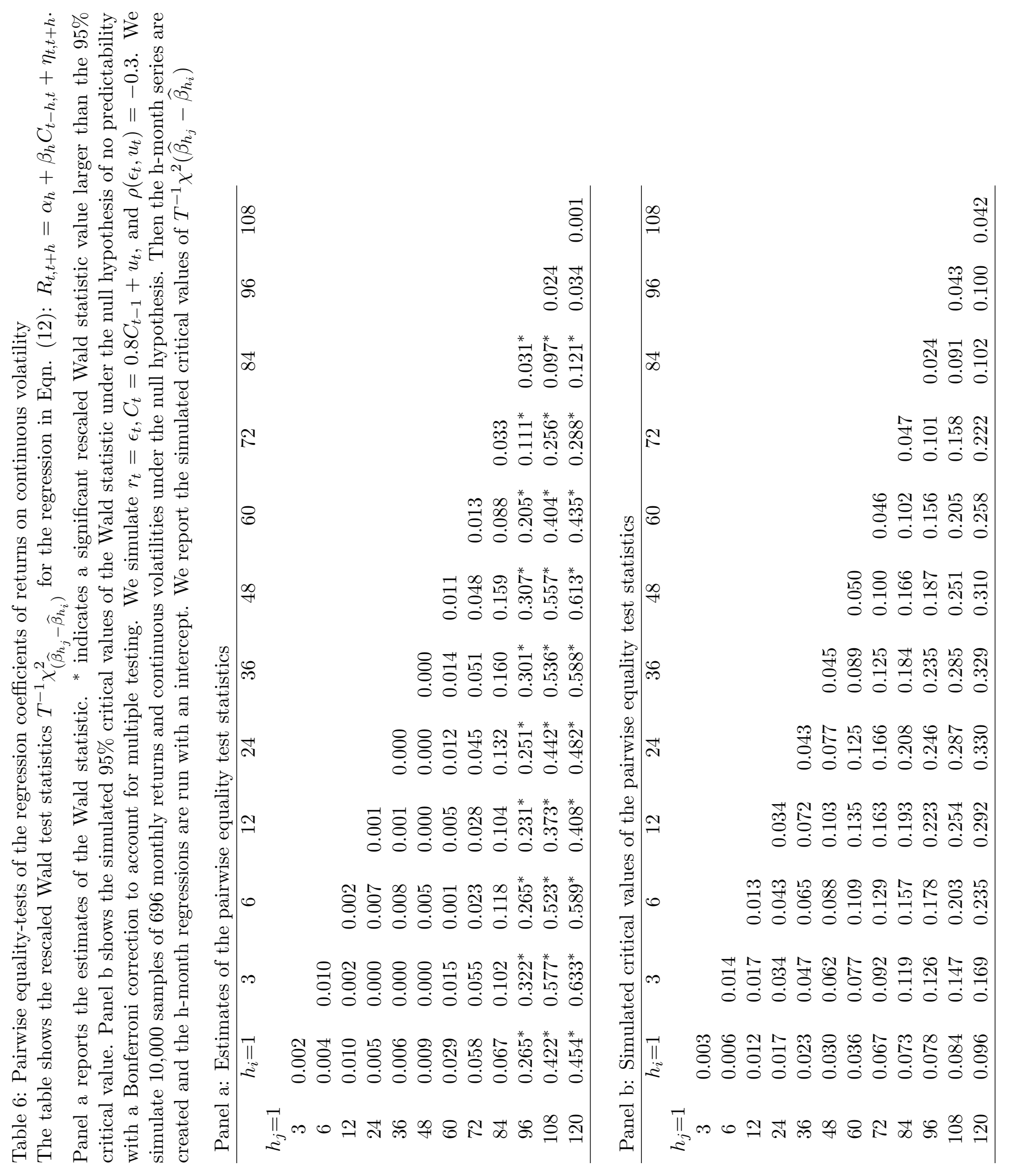


Table 7: pairwise equality test statistics - regressions with no intercept

The table shows the rescaled Wald test statistics $T^{-1} \chi_{\left(\widehat{\beta}_{h_{j}}-\widehat{\beta}_{h_{i}}\right)}^{2}$ for the regression in Eqn. (12): $R_{t, t+h}=\beta_{h} C_{t-h, t}+\eta_{t, t+h}$ run with no intercept. Panel a reports the estimates of the Wald statistic. ${ }^{*}$ indicates a significant rescaled Wald statistic value larger than the $95 \%$ critical value. Panel b reports the estimates of the Wald statistic for regressions run with no intercept and $99 \%$ winsorized continuous volatility.

\begin{tabular}{rcccccccccccc}
\multicolumn{1}{c}{ Panel a: No intercept $\left(\alpha_{h}=0\right)$} & \multicolumn{10}{c}{ (1) } \\
\hline & $h_{i}=1$ & 3 & 6 & 12 & 24 & 36 & 48 & 60 & 72 & 84 & 96 & 108 \\
$h_{j}=1$ & & & & & & & & & & & & \\
3 & 0.004 & & & & & & & & & & & \\
6 & $0.022^{*}$ & $0.010^{*}$ & & & & & & & & & & \\
12 & $0.028^{*}$ & $0.018^{*}$ & 0.002 & & & & & & & & & \\
24 & $0.039^{*}$ & $0.030^{*}$ & 0.005 & 0.001 & & & & & & & & \\
36 & $0.047^{*}$ & $0.041^{*}$ & 0.010 & 0.002 & 0.000 & & & & & & & \\
48 & $0.054^{*}$ & $0.049^{*}$ & 0.015 & 0.004 & 0.001 & 0.001 & & & & & & \\
60 & $0.064^{*}$ & $0.071^{*}$ & 0.026 & 0.010 & 0.006 & 0.006 & 0.005 & & & & & \\
72 & $0.075^{*}$ & $0.082^{*}$ & 0.038 & 0.015 & 0.015 & 0.015 & 0.016 & 0.005 & & & & \\
84 & $0.083^{*}$ & $0.096^{*}$ & 0.050 & 0.021 & 0.019 & 0.025 & 0.025 & 0.012 & 0.003 & & & \\
96 & $0.087^{*}$ & $0.108^{*}$ & 0.056 & 0.025 & 0.023 & 0.027 & 0.025 & 0.016 & 0.005 & 0.001 & & \\
108 & $0.090^{*}$ & $0.121^{*}$ & 0.058 & 0.026 & 0.025 & 0.029 & 0.027 & 0.016 & 0.006 & 0.002 & 0.000 \\
120 & $0.093^{*}$ & $0.136^{*}$ & 0.063 & 0.028 & 0.028 & 0.033 & 0.032 & 0.016 & 0.008 & 0.004 & 0.002 & 0.001 \\
\hline
\end{tabular}

Panel b: No intercept $\left(\alpha_{h}=0\right)$ - winsorized continuous volatility

\begin{tabular}{ccccccccccccc}
\hline & $h_{i}=1$ & 3 & 6 & 12 & 24 & 36 & 48 & 60 & 72 & 84 & 96 & 108 \\
$h_{j}=1$ & & & & & & & & & & & & \\
3 & 0.000 & & & & & & & & & & & \\
6 & 0.000 & 0.000 & & & & & & & & & & \\
12 & 0.000 & 0.001 & 0.000 & & & & & & & & & \\
24 & 0.000 & 0.001 & 0.000 & 0.000 & & & & & & & & \\
36 & 0.000 & 0.000 & 0.000 & 0.000 & 0.000 & & & & & & & \\
48 & 0.000 & 0.000 & 0.000 & 0.001 & 0.001 & 0.001 & & & & & & \\
60 & 0.001 & 0.000 & 0.001 & 0.004 & 0.007 & 0.007 & 0.006 & & & & & \\
72 & 0.002 & 0.001 & 0.005 & 0.011 & 0.019 & 0.020 & 0.022 & 0.009 & & & & \\
84 & 0.002 & 0.001 & 0.005 & 0.012 & 0.018 & 0.025 & 0.024 & 0.010 & 0.000 & & & \\
96 & 0.003 & 0.001 & 0.006 & 0.014 & 0.021 & 0.026 & 0.023 & 0.013 & 0.001 & 0.001 & & \\
108 & 0.003 & 0.002 & 0.006 & 0.014 & 0.024 & 0.028 & 0.025 & 0.013 & 0.002 & 0.001 & 0.000 & \\
120 & 0.003 & 0.002 & 0.007 & 0.016 & 0.026 & 0.031 & 0.029 & 0.013 & 0.003 & 0.003 & 0.001 & 0.000 \\
\hline
\end{tabular}


Table 8: Simulated critical values of the rescaled Wald statistic for regressions with no intercept. We simulate returns and volatilities under the null hypothesis of no predictability. The simulation setup is $r_{t}=\epsilon_{t}$, and $C_{t}=0.8 C_{t-1}+u_{t}, \operatorname{corr}\left(\epsilon_{t}, u_{t}\right)=-0.3$. We simulate 10,000 samples of 696 months of continuously compounded excess returns and continuous volatilities under the assumption of no predictability. The simulated series are aggregated over $h$ months (from 1 to 120 ) and the multi-period regressions are run without intercept. The table reports the critical value of the distribution of $T^{-1} \chi_{\left(\widehat{\beta}_{h_{j}}-\widehat{\beta}_{h_{i}}\right)}^{2}$ for a $5 \%$ test using a Bonferroni correction to account for multiple testing.

\begin{tabular}{ccccccccccccc}
\hline & $h_{i}=1$ & 3 & 6 & 12 & 24 & 36 & 48 & 60 & 72 & 84 & 96 & 108 \\
$h_{j}=1$ & & & & & & & & & & & & \\
3 & 0.005 & & & & & & & & & & & \\
6 & 0.006 & 0.004 & & & & & & & & & & \\
12 & 0.010 & 0.010 & 0.013 & & & & & & & & & \\
24 & 0.016 & 0.023 & 0.042 & 0.032 & & & & & & & & \\
36 & 0.022 & 0.034 & 0.062 & 0.067 & 0.038 & & & & & & & \\
48 & 0.027 & 0.042 & 0.081 & 0.094 & 0.068 & 0.041 & & & & & & \\
60 & 0.034 & 0.063 & 0.099 & 0.120 & 0.109 & 0.077 & 0.040 & & & & & \\
72 & 0.041 & 0.077 & 0.119 & 0.144 & 0.143 & 0.106 & 0.080 & 0.041 & & & & \\
84 & 0.048 & 0.085 & 0.139 & 0.170 & 0.175 & 0.152 & 0.123 & 0.085 & 0.040 & & & \\
96 & 0.056 & 0.094 & 0.160 & 0.198 & 0.209 & 0.194 & 0.155 & 0.127 & 0.083 & 0.037 & & \\
108 & 0.064 & 0.109 & 0.179 & 0.223 & 0.246 & 0.237 & 0.204 & 0.165 & 0.128 & 0.080 & 0.036 & \\
120 & 0.073 & 0.117 & 0.202 & 0.252 & 0.278 & 0.272 & 0.247 & 0.199 & 0.168 & 0.124 & 0.077 & 0.035 \\
\hline
\end{tabular}




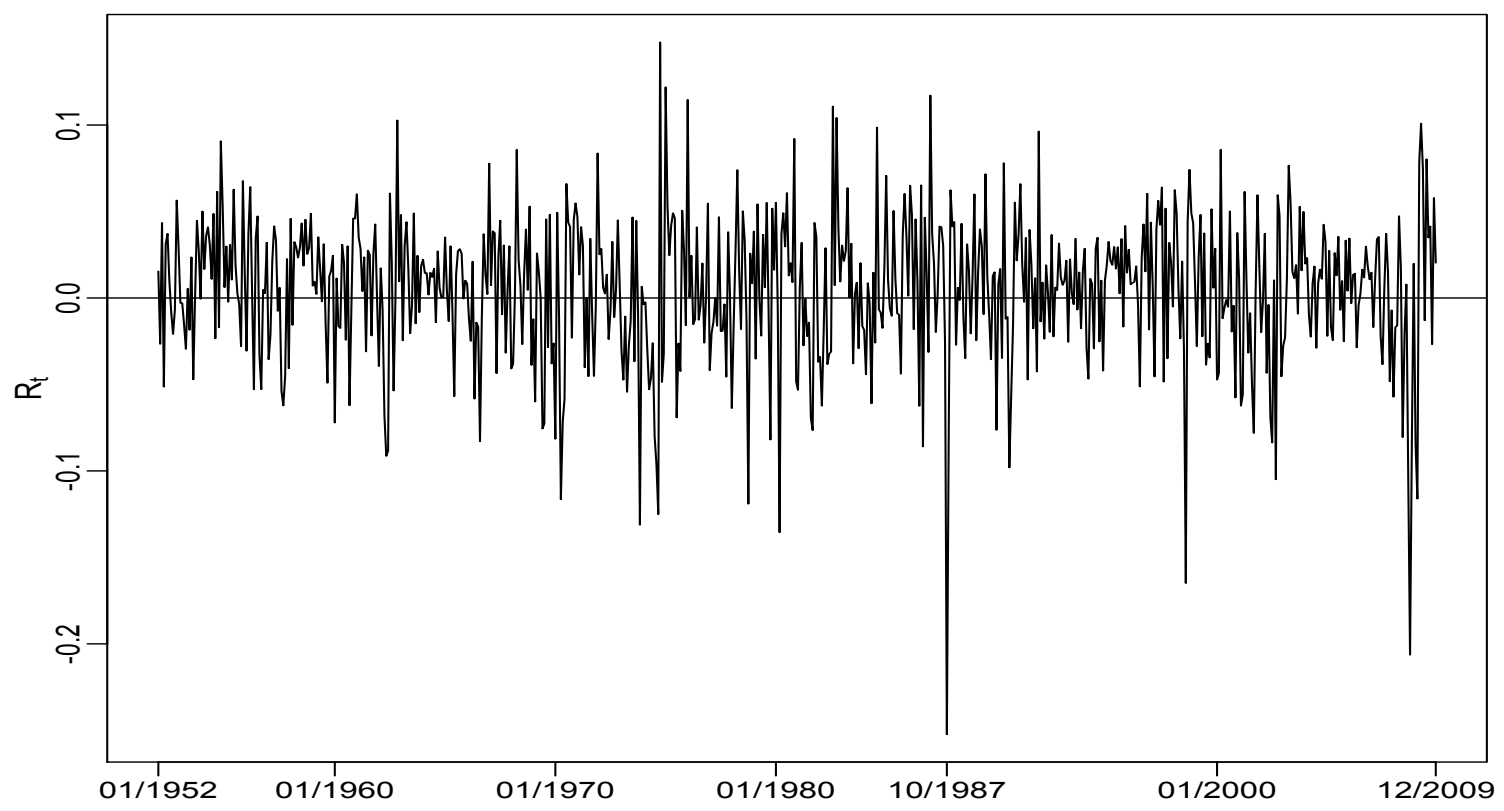

Figure 1: Monthly NYSE/AMEX excess return 1952-2009 

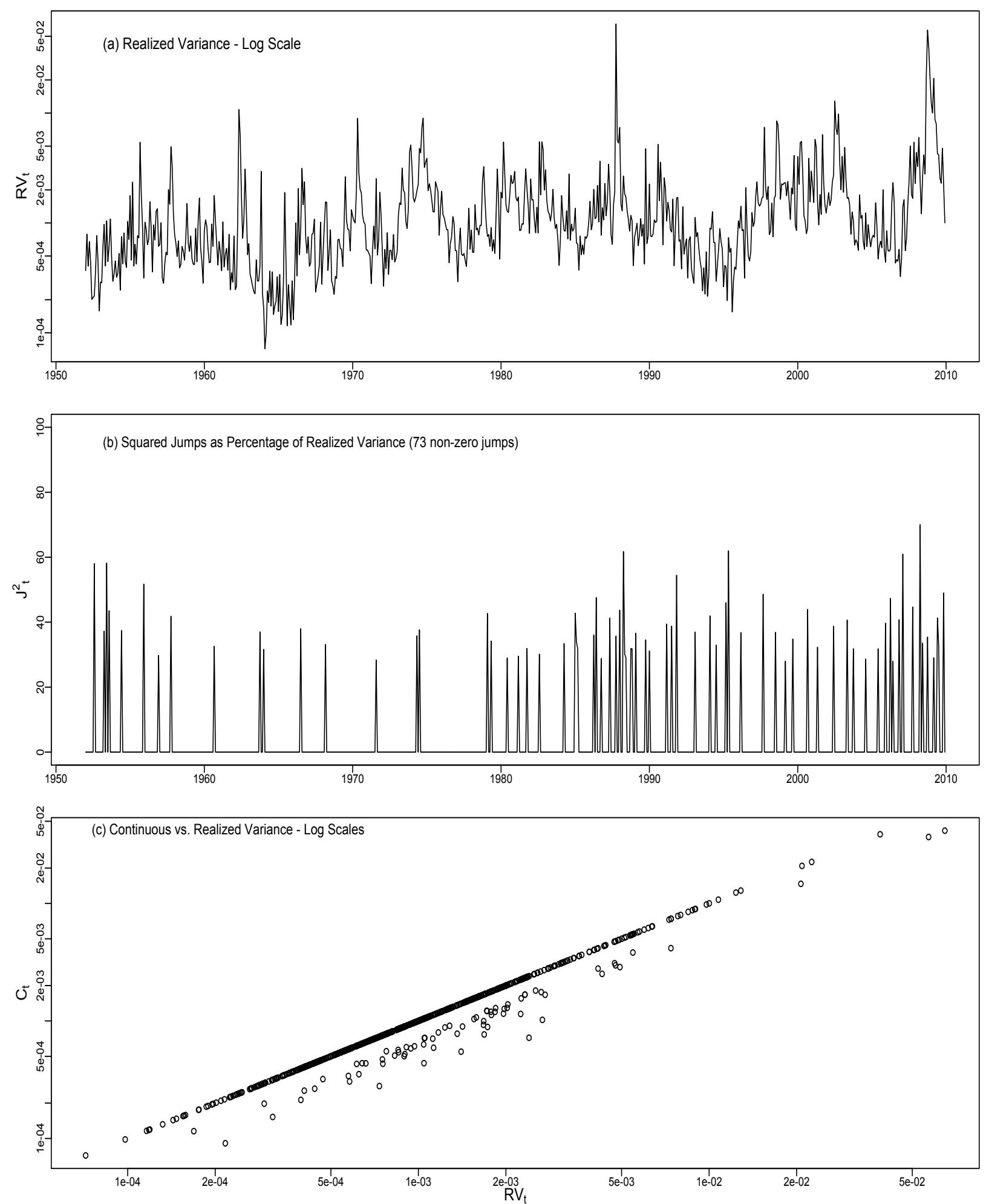

Figure 2: Monthly NYSE / AMEX realized variance, continuous volatility and squared jumps, from Jan. 52 to Dec. 09.

The significance level for the jump test statistic is 0.95 . 


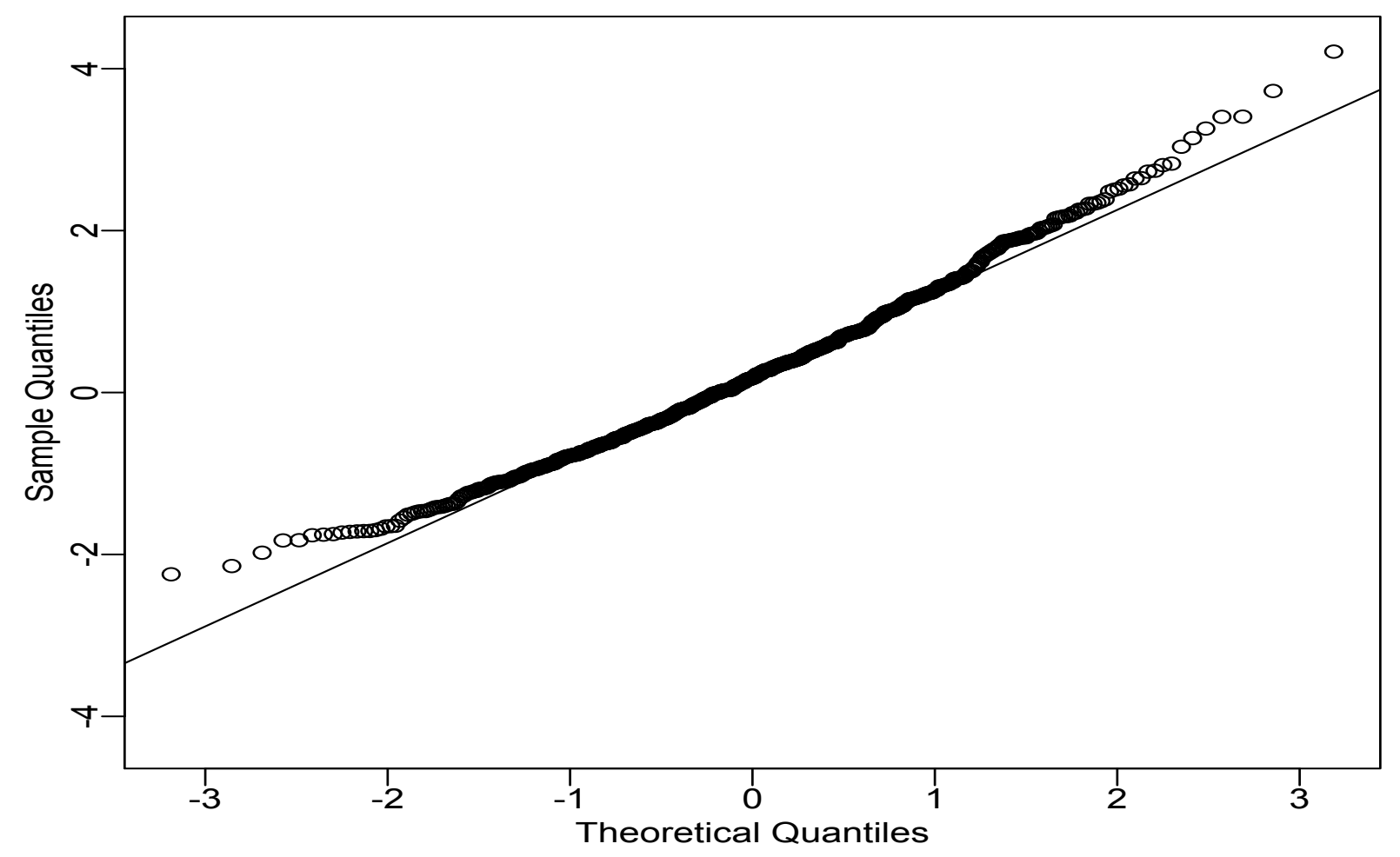

Figure 3: Normal probability plot for the jump test statistic. 

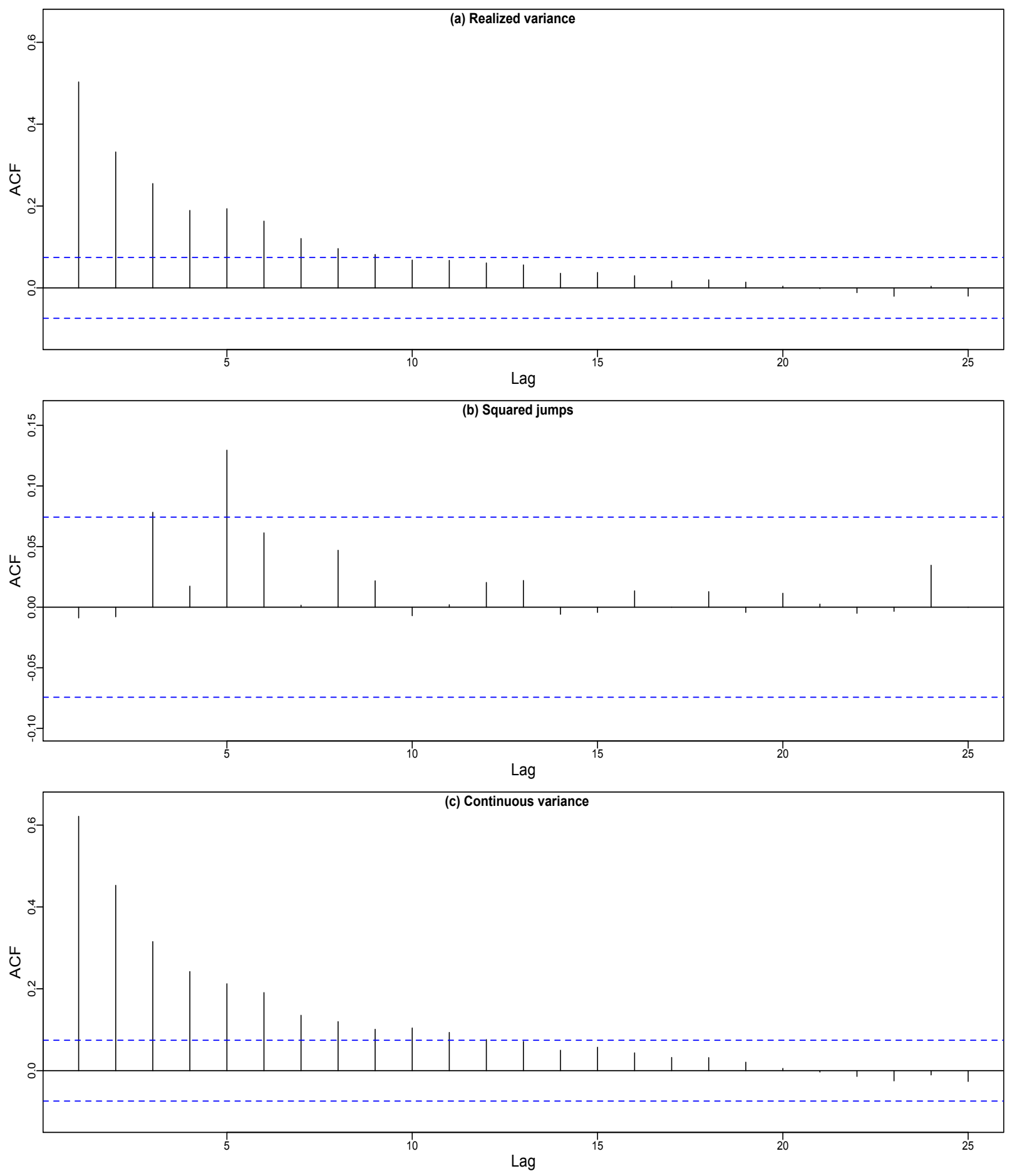

Figure 4: Autocorrelation of realized variance, continuous volatility and squared jumps. The significance level for the jump test statistic is 0.95 , see Figure 5 for a robustness analysis. 

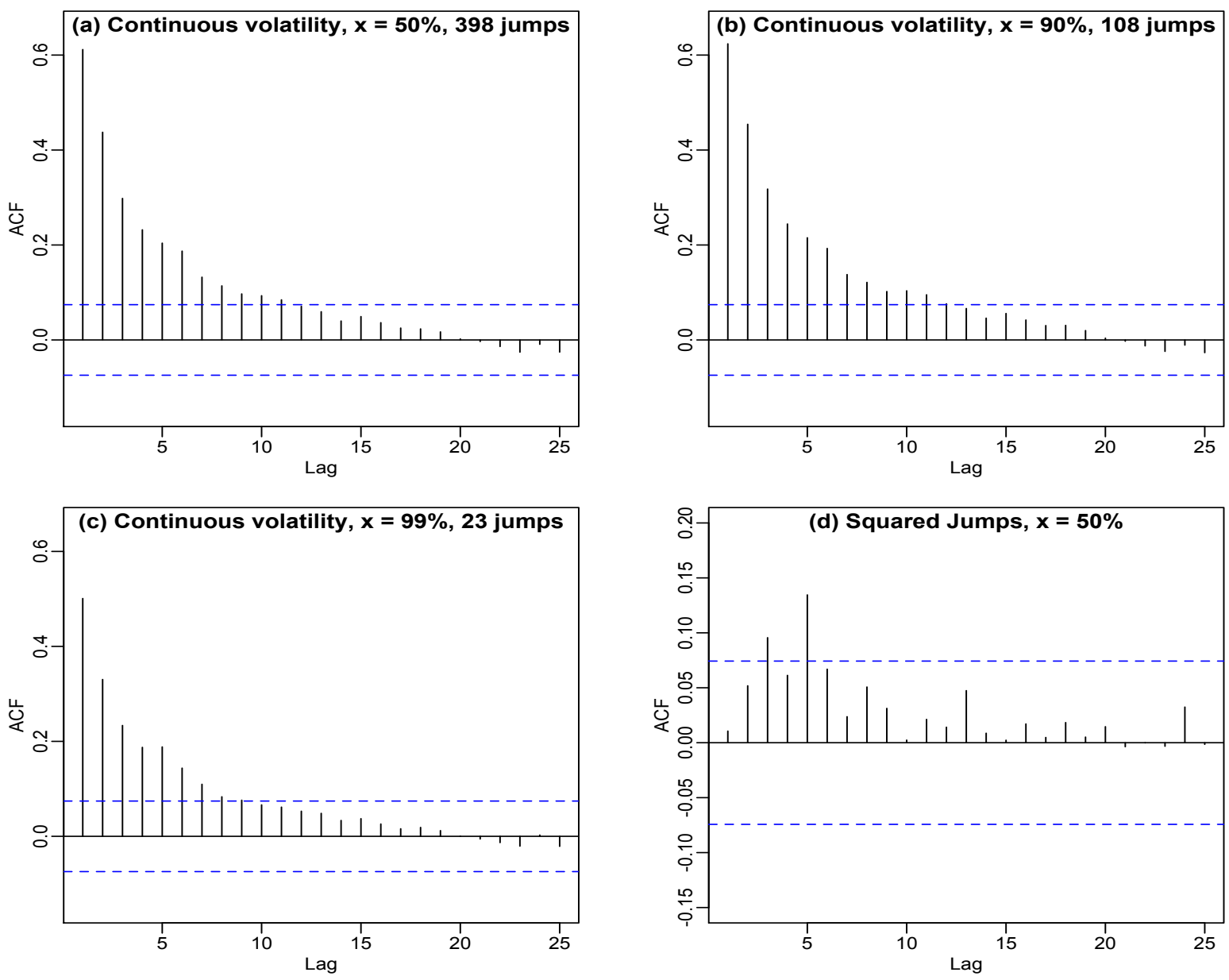

Figure 5: Robustness of the autocorrelation of continuous volatility to the significance level $x$. Panels (a)-(c): autocorrelation function of continuous volatility for significance levels of the jump test statistic equal to $0.5,0.9$, and 0.99. Panel (d): ACF of the jump process for the 0.5 significance level. 


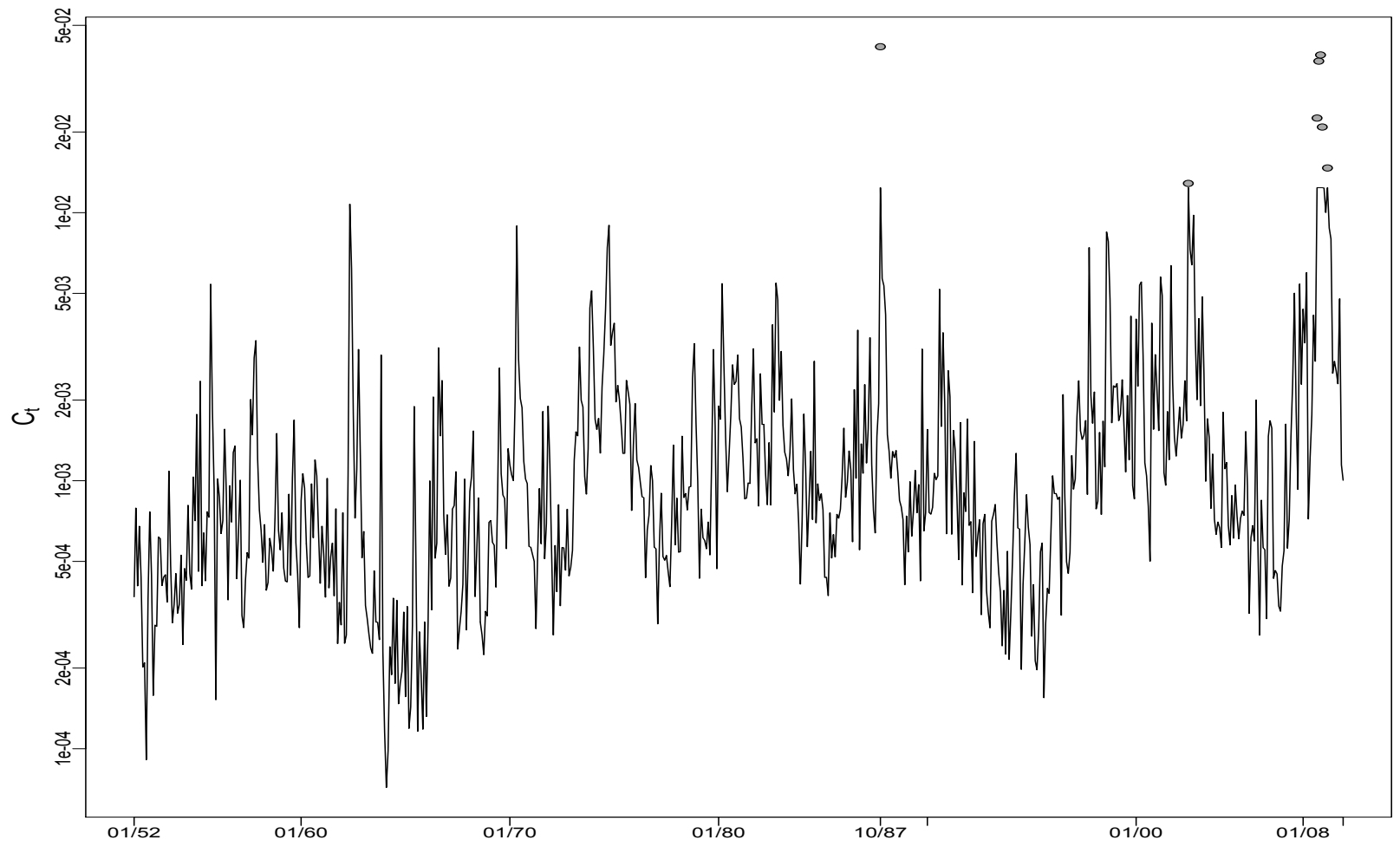

Figure 6: Continuous volatility with winsorized top $1 \%$ values, Log-Scale.

Continuous volatility estimated with $95 \%$ significance level of the $z$ statistic. The circles are the winsorized top $1 \%$ larger values 


\section{APPENDIX A: Propositions}

Proposition 1 (Unrestricted regressions). For dynamics in Eqn.(11), $b_{1}=\gamma_{1}=0$, and Eqn. (1) estimated for any generic horizon $h$, we have:

$T\left(\begin{array}{c}\widehat{b}_{h} \\ \widehat{\gamma}_{h}\end{array}\right) \stackrel{\mathcal{L}}{\longrightarrow}\left[\begin{array}{c}\frac{\sigma_{e}}{\sigma_{u} C} \frac{\int_{\lambda}^{1-\lambda} \bar{J}_{c_{2}}^{2}(s,-\lambda) d s \int_{\lambda}^{1-\lambda} \bar{W}(s, \lambda) \bar{J}_{c_{1}}(s,-\lambda) d s-\int_{\lambda}^{1-\lambda} \bar{J}_{c_{1}}(s,-\lambda) \bar{J}_{c_{2}}(s,-\lambda) d s \int_{\lambda}^{1-\lambda} \bar{W}(s, \lambda) \bar{J}_{c_{2}}(s,-\lambda) d s}{\int_{\lambda}^{1-\lambda} \bar{J}_{c_{1}}^{2}(s,-\lambda) d s \int_{\lambda}^{1-\lambda} \bar{J}_{c_{2}}^{2}(s,-\lambda) d s-\left(\int_{\lambda}^{1-\lambda} \bar{J}_{c_{1}}(s,-\lambda) \bar{J}_{c_{2}}(s,-\lambda) d s\right)^{2}} \\ \frac{\sigma_{e}}{\sigma_{u \mathcal{J}^{2}}} \frac{\int_{\lambda}^{1-\lambda} \bar{J}_{c_{1}}^{2}(s,-\lambda) d s \int_{\lambda}^{1-\lambda} \bar{W}(s, \lambda) \bar{J}_{c_{2}}(s,-\lambda) d s-\int_{\lambda}^{1-\lambda} \bar{J}_{c_{1}}(s,-\lambda) \bar{J}_{c_{2}}(s,-\lambda) d s \int_{\lambda}^{1-\lambda} \bar{W}(s, \lambda) \bar{J}_{c_{1}}(s,-\lambda) d s}{\int_{\lambda}^{1-\lambda} \bar{J}_{c_{1}}^{2}(s,-\lambda) d s \int_{\lambda}^{1-\lambda} \bar{J}_{c_{2}}^{2}(s,-\lambda) d s-\left(\int_{\lambda}^{1-\lambda} \bar{J}_{c_{1}}(s,-\lambda) \bar{J}_{c_{2}}(s,-\lambda) d s\right)^{2}}\end{array}\right]$,

and

$T^{3} \operatorname{Var}\left(\begin{array}{c}\widehat{b}_{h} \\ \widehat{\gamma}_{h}\end{array}\right) \stackrel{\mathcal{L}}{\longrightarrow} \frac{\bar{\Omega}}{(1-2 \lambda) \bar{\Delta}}$

$\times\left[\begin{array}{cc}\frac{\sigma_{e}^{2}}{\sigma_{u}^{2} C} \int_{\lambda}^{1-\lambda} \bar{J}_{c_{2}}^{2}(s,-\lambda) d s & \frac{-\sigma_{e}^{2}}{\sigma_{u} \sigma_{u} \mathcal{J}^{2}} \int_{\lambda}^{1-\lambda} \bar{J}_{c_{1}}(s,-\lambda) \bar{J}_{c_{2}}(s,-\lambda) d s \\ \frac{-\sigma_{e}^{2}}{\sigma_{u} \sigma^{\sigma}{ }_{u} \mathcal{J}^{2}} \int_{\lambda}^{1-\lambda} \bar{J}_{c_{1}}(s,-\lambda) \bar{J}_{c_{2}}(s,-\lambda) d s & \frac{\sigma_{e}^{2}}{\sigma_{u}^{2} \mathcal{J}^{2}} \int_{\lambda}^{1-\lambda} \bar{J}_{c_{1}}^{2}(s,-\lambda) d s\end{array}\right]$,

where $\bar{\Delta}=\int_{\lambda}^{1-\lambda} \bar{J}_{c_{1}}^{2}(s,-\lambda) d s \int_{\lambda}^{1-\lambda} \bar{J}_{c_{2}}^{2}(s,-\lambda) d s-\left(\int_{\lambda}^{1-\lambda} \bar{J}_{c_{1}}(s,-\lambda) \bar{J}_{c_{2}}(s,-\lambda) d s\right)^{2}$,

$\bar{\Omega}=\left[\begin{array}{c}\int_{\lambda}^{1-\lambda} \bar{W}^{2}(s, \lambda) d s \\ -\bar{\Delta}^{-1} \int_{\lambda}^{1-\lambda} \bar{J}_{c_{2}}^{2}(s,-\lambda) d s\left(\int_{\lambda}^{1-\lambda} \bar{W}(s, \lambda) \bar{J}_{c_{1}}(s,-\lambda) d s\right)^{2} \\ -\bar{\Delta}^{-1} \int_{\lambda}^{1-\lambda} \bar{J}_{c_{1}}^{2}(s,-\lambda) d s\left(\int_{\lambda}^{1-\lambda} \bar{W}(s, \lambda) \bar{J}_{c_{2}}(s,-\lambda) d s\right)^{2} \\ +2 \bar{\Delta}^{-1} \int_{\lambda}^{1-\lambda} \bar{W}(s, \lambda) \bar{J}_{c_{1}}(s,-\lambda) d s \int_{\lambda}^{1-\lambda} \bar{J}_{c_{1}}(s,-\lambda) \bar{J}_{c_{2}}(s,-\lambda) d s \int_{\lambda}^{1-\lambda} \bar{W}(s, \lambda) \bar{J}_{c_{2}}(s,-\lambda) d s\end{array}\right]$,

$\bar{W}(t, \lambda)=\{W(t+\lambda)-W(t)\}-\frac{1}{1-2 \lambda} \int_{\lambda}^{1-\lambda}(W(s+\lambda)-W(s)) d s$,

$\bar{J}_{c_{i}}(t,-\lambda)=\left\{\int_{t-\lambda}^{t} J_{c_{i}}(s) d s\right\}-\frac{1}{1-2 \lambda} \int_{\lambda}^{1-\lambda}\left(\int_{t-\lambda}^{t} J_{c_{i}}(s) d s\right) d t, i=1,2$,

$d J_{c_{i}}(s)=c_{i} J_{c_{i}}(s)+d B_{i}(s), J_{c_{i}}(0)=0$ or $J_{c_{i}}(s)=B_{i}(s)+c_{i} \int_{0}^{s} e^{c_{i}(s-\tau)} B_{i}(\tau) d \tau, i=1,2$,

and $\left\{W(s), B_{1}(s), B_{2}(s)\right\}$ is a vector of standard Brownian motions with covariance

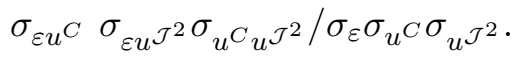

Proposition 2 (The restricted regressions). For dynamics as in Eqn. (11), $b_{1}=\gamma_{1}=0$, and Eqn.

(1) estimated with $\alpha_{h}=0$ for any generic horizon $h$, we have: 
$T\left(\begin{array}{c}\widehat{b}_{h} \\ \widehat{\gamma}_{h}\end{array}\right) \stackrel{\mathcal{L}}{\longrightarrow}\left[\begin{array}{c}\frac{\sigma_{\varepsilon}}{\sigma_{u} C} \frac{\int_{\lambda}^{1-\lambda} J_{c_{2}}^{2}(s,-\lambda) d s \int_{\lambda}^{1-\lambda} W(s, \lambda) J_{c_{1}}(s,-\lambda) d s-\int_{\lambda}^{1-\lambda} J_{c_{1}}(s,-\lambda) J_{c_{2}}(s,-\lambda) d s \int_{\lambda}^{1-\lambda} W(s, \lambda) J_{c_{2}}(s,-\lambda) d s}{\int_{\lambda}^{1-\lambda} J_{c_{1}}^{2}(s,-\lambda) d s \int_{\lambda}^{1-\lambda} J_{c_{2}}^{2}(s,-\lambda) d s-\left(\int_{\lambda}^{1-\lambda} J_{c_{1}}(s,-\lambda) J_{c_{2}}(s,-\lambda) d s\right)^{2}} \\ \frac{\sigma_{\varepsilon}}{\sigma_{u} \mathcal{J}^{2}} \frac{\int_{\lambda}^{1-\lambda} J_{c_{1}}^{2}(s,-\lambda) d s \int_{\lambda}^{1-\lambda} W(s, \lambda) J_{c_{2}}(s,-\lambda) d s-\int_{\lambda}^{1-\lambda} J_{c_{1}}(s,-\lambda) J_{c_{2}}(s,-\lambda) d s \int_{\lambda}^{1-\lambda} W(s, \lambda) J_{c_{1}}(s,-\lambda) d s}{\int_{\lambda}^{1-\lambda} J_{c_{1}}^{2}(s,-\lambda) d s \int_{\lambda}^{1-\lambda} J_{c_{2}}^{2}(s,-\lambda) d s-\left(\int_{\lambda}^{1-\lambda} J_{c_{1}}(s,-\lambda) J_{c_{2}}(s,-\lambda) d s\right)^{2}}\end{array}\right]$,

and

$T^{3} \operatorname{Var}\left(\begin{array}{c}\widehat{b}_{h} \\ \widehat{\gamma}_{h}\end{array}\right) \stackrel{\mathcal{L}}{\longrightarrow} \frac{\Omega}{(1-2 \lambda) \Delta}\left[\begin{array}{cc}\frac{\sigma_{e}^{2}}{\sigma_{u}^{2}} \int_{\lambda}^{1-\lambda} J_{c_{2}}^{2}(s,-\lambda) d s & \frac{-\sigma_{e}^{2}}{\sigma_{u} \sigma_{u} \mathcal{J}^{2}} \int_{\lambda}^{1-\lambda} J_{c_{1}}(s,-\lambda) J_{c_{2}}(s,-\lambda) d s \\ \frac{-\sigma_{e}^{2}}{\sigma_{u} \sigma_{u \mathcal{J}^{2}}} \int_{\lambda}^{1-\lambda} J_{c_{1}}(s,-\lambda) J_{c_{2}}(s,-\lambda) d s & \frac{\sigma_{e}^{2}}{\sigma_{u}^{2} \mathcal{J}^{2}} \int_{\lambda}^{1-\lambda} J_{c_{1}}^{2}(s,-\lambda) d s\end{array}\right]$, where

$\Delta=\int_{\lambda}^{1-\lambda} J_{c_{1}}^{2}(s,-\lambda) d s \int_{\lambda}^{1-\lambda} J_{c_{2}}^{2}(s,-\lambda) d s-\left(\int_{\lambda}^{1-\lambda} J_{c_{1}}(s,-\lambda) J_{c_{2}}(s,-\lambda) d s\right)^{2}$,

$\Omega=\left[\begin{array}{c}\int_{\lambda}^{1-\lambda} W^{2}(s, \lambda) d s \\ -\Delta^{-1} \int_{\lambda}^{1-\lambda} J_{c_{2}}^{2}(s,-\lambda) d s\left(\int_{\lambda}^{1-\lambda} W(s, \lambda) J_{c_{1}}(s,-\lambda) d s\right)^{2} \\ -\Delta^{-1} \int_{\lambda}^{1-\lambda} J_{c_{1}}^{2}(s,-\lambda) d s\left(\int_{\lambda}^{1-\lambda} W(s, \lambda) J_{c_{2}}(s,-\lambda) d s\right)^{2} \\ +2 \Delta^{-1} \int_{\lambda}^{1-\lambda} W(s, \lambda) J_{c_{1}}(s,-\lambda) d s \int_{\lambda}^{1-\lambda} J_{c_{1}}(s,-\lambda) J_{c_{2}}(s,-\lambda) d s \int_{\lambda}^{1-\lambda} W(s, \lambda) J_{c_{2}}(s,-\lambda) d s\end{array}\right]$,

$W(t, \lambda)=W(t+\lambda)-W(t)$,

$J_{c_{i}}(t,-\lambda)=\int_{t-\lambda}^{t} J_{c_{i}}(s) d s, i=1,2$,

$d J_{c_{i}}(s)=c_{i} J_{c_{i}}(s)+d B_{i}(s), J_{c_{i}}(0)=0$ or $J_{c_{i}}(s)=B_{i}(s)+c_{i} \int_{0}^{s} e^{c_{i}(s-\tau)} B_{i}(\tau) d \tau, i=1,2$,

and $\left\{W(s), B_{1}(s), B_{2}(s)\right\}$ is a vector of standard Brownian motions with covariance

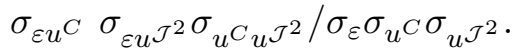

Proposition 3 (The unrestricted regressions). For dynamics Eqn. (13) and Eqn. (14), $\beta_{1}=0$, and Eqn. (12) estimated for any two generic levels of aggregation $h_{i}$ and $h_{j}$ (w.o.l.g), we have:

$T\left(\begin{array}{l}\widehat{\beta}_{h_{i}} \\ \widehat{\beta}_{h_{j}}\end{array}\right) \stackrel{\mathcal{L}}{\longrightarrow} \frac{\sigma_{\varepsilon}}{\sigma_{u}}\left[\begin{array}{c}\frac{\int_{\lambda_{i}}^{1-\lambda_{i}} \bar{W}\left(s, \lambda_{i}\right) \bar{J}_{c}\left(s,-\lambda_{i}\right) d s}{\int_{\lambda_{i}}^{1-\lambda_{i}} \bar{J}_{c}^{2}\left(s,-\lambda_{i}\right) d s} \\ \frac{\int_{\lambda_{j}}^{1-\lambda_{j}} \bar{W}\left(s, \lambda_{j}\right) \bar{J}_{c}\left(s,-\lambda_{j}\right) d s}{\int_{\lambda_{j}}^{1-\lambda_{j}} \bar{J}_{c}^{2}\left(s,-\lambda_{j}\right) d s}\end{array}\right]$,

$T^{3} \operatorname{Var}\left(\begin{array}{c}\widehat{\beta}_{h_{i}} \\ \widehat{\beta}_{h_{j}}\end{array}\right) \stackrel{\mathcal{L}}{\longrightarrow}\left[\begin{array}{cc}\overline{\operatorname{Avar}}\left(\widehat{\beta}_{h_{i}}\right) & \overline{\operatorname{Acov}}\left(\widehat{\beta}_{h_{i}}, \widehat{\beta}_{h_{j}}\right) \\ \overline{\operatorname{Acov}}\left(\widehat{\beta}_{h_{i}}, \widehat{\beta}_{h_{j}}\right) & \overline{\operatorname{Avar}}\left(\widehat{\beta}_{h_{j}}\right)\end{array}\right]$

where 


$$
\begin{aligned}
& \overline{A v a r}\left(\widehat{\beta}_{h_{l}}\right)=\frac{\sigma_{\varepsilon}^{2}}{\sigma_{u}^{2}} \frac{1}{1-2 \lambda_{l}} \frac{\left[\int_{\lambda_{l}}^{1-\lambda_{l}} \bar{W}^{2}\left(s, \lambda_{l}\right) d s \int_{\lambda_{l}}^{1-\lambda_{l}} \bar{J}_{c}^{2}\left(s,-\lambda_{l}\right) d s-\left(\int_{\lambda_{l}}^{1-\lambda_{l}} \bar{W}\left(s, \lambda_{l}\right) \bar{J}_{c}\left(s,-\lambda_{l}\right) d s\right)^{2}\right]}{\left(\int_{\lambda_{l}}^{1-\lambda_{l}} \bar{J}_{c}^{2}\left(s,-\lambda_{l}\right) d s\right)^{2}}, l=i, j,
\end{aligned}
$$

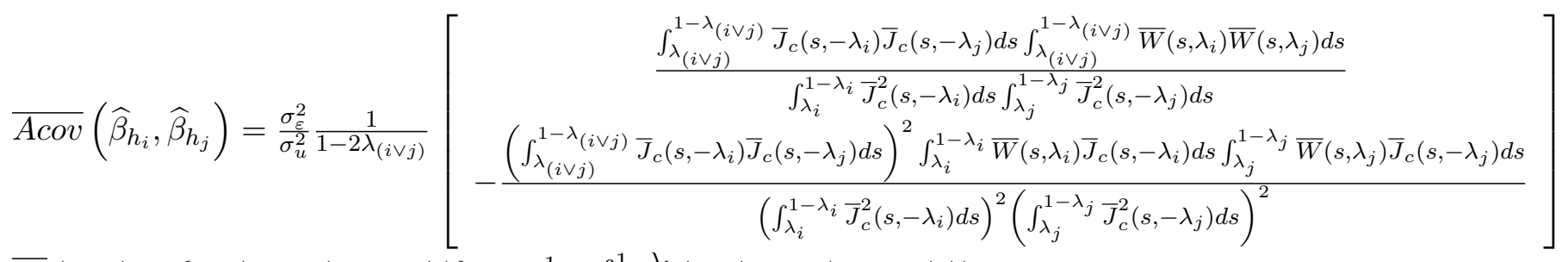

$$
\begin{aligned}
& \bar{W}\left(t, \lambda_{l}\right)=\left\{W\left(t+\lambda_{l}\right)-W(t)\right\}-\frac{1}{1-2 \lambda_{l}} \int_{\lambda_{l}}^{1-\lambda_{l}}\left(W\left(s+\lambda_{l}\right)-W(s)\right) d s, l=i, j, \\
& \bar{J}_{c}\left(t,-\lambda_{l}\right)=\left\{\int_{t-\lambda_{l}}^{t} J_{c}(s) d s\right\}-\frac{1}{1-2 \lambda_{l}} \int_{\lambda_{l}}^{1-\lambda_{l}}\left(\int_{t-\lambda_{l}}^{t} J_{c}(s) d s\right) d t, l=i, j, \\
& d J_{c}(s)=c J_{c}(s)+d B(s), J_{c}(0)=0 \text { or } J_{c}(s)=B(s)+c \int_{0}^{s} e^{c(s-\tau)} B(\tau) d \tau, \\
& h_{(i \vee j)} \equiv \max \left(h_{i}, h_{j}\right) \text { and }\{W(s), B(s)\} \text { is a vector of standard Brownian motions with covariance } \\
& \sigma_{\varepsilon u} / \sigma_{\varepsilon} \sigma_{u} \text {. }
\end{aligned}
$$

Proposition 4 (The restricted regressions). For dynamics Eqn. (13) and Eqn. (14), $\beta_{1}=0$, and Eqn. (12) estimated with $\alpha_{h}=0$ for any two generic levels of aggregation $h_{i}$ and $h_{j}$ (w.o.l.g), we have:

$T\left(\begin{array}{l}\widehat{\beta}_{h_{i}} \\ \widehat{\beta}_{h_{j}}\end{array}\right) \stackrel{\mathcal{L}}{\longrightarrow} \frac{\sigma_{\varepsilon}}{\sigma_{u}}\left[\begin{array}{c}\frac{\int_{\lambda_{i}}^{1-\lambda_{i}} W\left(s, \lambda_{i}\right) J_{c}\left(s,-\lambda_{i}\right) d s}{\int_{\lambda_{i}}^{1-\lambda_{i}} J_{c}^{2}\left(s,-\lambda_{i}\right) d s} \\ \frac{\int_{\lambda_{j}}^{1-\lambda_{j}} W\left(s, \lambda_{j}\right) J_{c}\left(s,-\lambda_{j}\right) d s}{\int_{\lambda_{j}}^{1-\lambda_{j}} J_{c}^{2}\left(s,-\lambda_{j}\right) d s}\end{array}\right]$,

$T^{3} \operatorname{Var}\left(\begin{array}{c}\widehat{\beta}_{h_{i}} \\ \widehat{\beta}_{h_{j}}\end{array}\right) \stackrel{\mathcal{L}}{\longrightarrow}\left[\begin{array}{cc}\operatorname{Avar}\left(\widehat{\beta}_{h_{i}}\right) & \operatorname{Acov}\left(\widehat{\beta}_{h_{i}}, \widehat{\beta}_{h_{j}}\right) \\ \operatorname{Acov}\left(\widehat{\beta}_{h_{i}}, \widehat{\beta}_{h_{j}}\right) & \operatorname{Avar}\left(\widehat{\beta}_{h_{j}}\right)\end{array}\right]$,

where

$\operatorname{Avar}\left(\widehat{\beta}_{h_{l}}\right)=\frac{\sigma_{\varepsilon}^{2}}{\sigma_{u}^{2}} \frac{1}{1-2 \lambda_{l}} \frac{\left[\int_{\lambda_{l}}^{1-\lambda_{l}} W^{2}\left(s, \lambda_{l}\right) d s \int_{\lambda_{l}}^{1-\lambda_{l}} J_{c}^{2}\left(s,-\lambda_{l}\right) d s-\left(\int_{\lambda_{l}}^{1-\lambda_{l}} W\left(s, \lambda_{l}\right) J_{c}\left(s,-\lambda_{l}\right) d s\right)^{2}\right]}{\left(\int_{\lambda_{l}}^{1-\lambda_{l}} J_{c}^{2}\left(s,-\lambda_{l}\right) d s\right)^{2}}, l=i, j$,

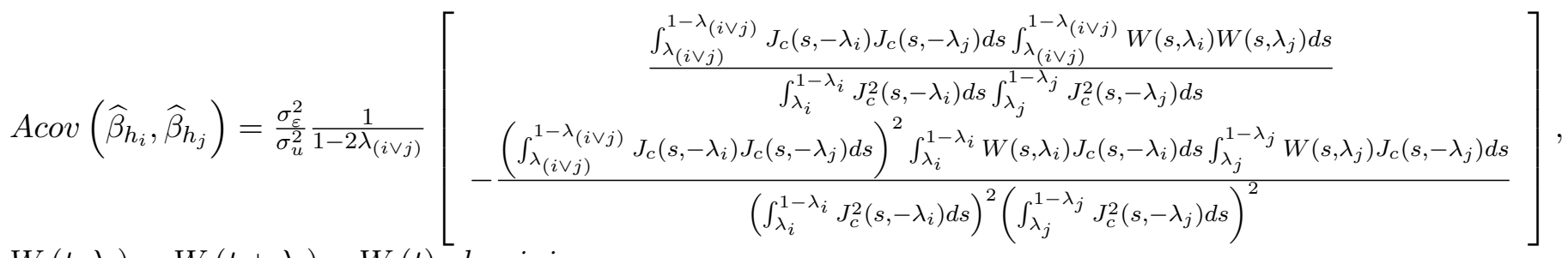

$W\left(t, \lambda_{l}\right)=W\left(t+\lambda_{l}\right)-W(t), l=i, j$ 
$J_{c}\left(t,-\lambda_{l}\right)=\int_{t-\lambda_{l}}^{t} J_{c}(s) d s, l=i, j$,

$d J_{c}(s)=c J_{c}(s)+d B(s), J_{c}(0)=0$ or $J_{c}(s)=B(s)+c \int_{0}^{s} e^{c(s-\tau)} B(\tau) d \tau$,

$h_{(i \vee j)} \equiv \max \left(h_{i}, h_{j}\right)$ and $\{W(s), B(s)\}$ is a vector of standard Brownian motions with covariance $\sigma_{\varepsilon u} / \sigma_{\varepsilon} \sigma_{u}$.

\section{APPENDIX B: Proofs}

\section{B1: $\quad$ Proof of Proposition 1}

Define $\overline{R_{h}}=\frac{1}{T-2 h+1} \sum_{t=1}^{T-2 h+1} R_{t, t+h}, \overline{C_{-h}}=\frac{1}{T-2 h+1} \sum_{t=1}^{T-2 h+1} C_{t-h, t}$, and $\overline{\mathcal{J}_{-h}^{2}}=\frac{1}{T-2 h+1} \sum_{t=1}^{T-2 h+1} \mathcal{J}_{t-h, t}^{2}$. Then:

1. $T\left(\begin{array}{c}\widehat{b}_{h} \\ \widehat{\gamma}_{h}\end{array}\right)=\left[\begin{array}{cc}\frac{1}{T} \sum_{t=1}^{T-2 h+1}\left(\frac{C_{t-h, t}-\overline{C_{-h}}}{T^{3 / 2}}\right)^{2} & \left.\frac{1}{T} \sum_{t=1}^{T-2 h+1}\left(\frac{C_{t-h, t}-\overline{C_{-h}}}{T^{3 / 2}}\right)\left(\frac{\mathcal{J}_{t-h, t}^{2}-\overline{\mathcal{J}_{-h}^{2}}}{T^{3 / 2}}\right)\right]^{-1} \\ \frac{1}{T} \sum_{t=1}^{T-2 h+1}\left(\frac{C_{t-h, t}-\overline{C_{-h}}}{T^{3 / 2}}\right)\left(\frac{\mathcal{J}_{t-h, t}^{2}-\overline{\mathcal{J}_{-h}^{2}}}{T^{3 / 2}}\right) & \frac{1}{T} \sum_{t=1}^{T-2 h+1}\left(\frac{\mathcal{J}_{t-h, t}^{2}-\overline{\mathcal{J}_{-h}^{2}}}{T^{3 / 2}}\right)^{2}\end{array}\right]^{2}$

$\times\left[\begin{array}{cc}\frac{1}{T} \sum_{t=1}^{T-2 h+1}\left(\frac{C_{t-h, t}-\overline{C_{-h}}}{T^{3 / 2}}\right) & \left(\frac{R_{t, t+h}-\overline{R_{h}}}{T^{1 / 2}}\right) \\ \frac{1}{T} \sum_{t=1}^{T-2 h+1}\left(\frac{\mathcal{J}_{t-h, t}^{2}-\overline{\mathcal{J}_{-h}^{2}}}{T^{3 / 2}}\right) & \left(\frac{R_{t, t+h}-\overline{R_{h}}}{T^{1 / 2}}\right)\end{array}\right]$

$\stackrel{\mathcal{L}}{\longrightarrow}\left[\begin{array}{c}\frac{\sigma_{e}}{\sigma_{u} C} \frac{\int_{\lambda}^{1-\lambda} \bar{J}_{c_{2}}^{2}(s,-\lambda) d s \int_{\lambda}^{1-\lambda} \bar{W}(s, \lambda) \bar{J}_{c_{1}}(s,-\lambda) d s-\int_{\lambda}^{1-\lambda} \bar{J}_{c_{1}}(s,-\lambda) \bar{J}_{c_{2}}(s,-\lambda) d s \int_{\lambda}^{1-\lambda} \bar{W}(s, \lambda) \bar{J}_{c_{2}}(s,-\lambda) d s}{\int_{\lambda}^{1-\lambda} \bar{J}_{c_{1}}^{2}(s,-\lambda) d s \int_{\lambda}^{1-\lambda} \bar{J}_{c_{2}}^{2}(s,-\lambda) d s-\left(\int_{\lambda}^{1-\lambda} \bar{J}_{c_{1}}(s,-\lambda) \bar{J}_{c_{2}}(s,-\lambda) d s\right)^{2}} \\ \frac{\sigma_{e}}{\sigma_{u \mathcal{J}^{2}}} \frac{\int_{\lambda}^{1-\lambda} \bar{J}_{c_{1}}^{2}(s,-\lambda) d s \int_{\lambda}^{1-\lambda} \bar{W}(s, \lambda) \bar{J}_{c_{2}}(s,-\lambda) d s-\int_{\lambda}^{1-\lambda} \bar{J}_{c_{1}}(s,-\lambda) \bar{J}_{c_{2}}(s,-\lambda) d s \int_{\lambda}^{1-\lambda} \bar{W}(s, \lambda) \bar{J}_{c_{1}}(s,-\lambda) d s}{\int_{\lambda}^{1-\lambda} \bar{J}_{c_{1}}^{2}(s,-\lambda) d s \int_{\lambda}^{1-\lambda} \bar{J}_{c_{2}}^{2}(s,-\lambda) d s-\left(\int_{\lambda}^{1-\lambda} \bar{J}_{c_{1}}(s,-\lambda) \bar{J}_{c_{2}}(s,-\lambda) d s\right)^{2}}\end{array}\right]$

2. $T^{3} \operatorname{Var}\left(\begin{array}{c}\widehat{b}_{h} \\ \hat{\gamma}_{h}\end{array}\right)=\frac{S^{2}}{T}\left[\begin{array}{cc}\frac{1}{T} \sum_{t=1}^{T-2 h+1}\left(\frac{C_{t-h, t}-\overline{C_{-h}}}{T^{3 / 2}}\right)^{2} & \frac{1}{T} \sum_{t=1}^{T-2 h+1}\left(\frac{C_{t-h, t}-\overline{C_{-h}}}{T^{3 / 2}}\right)\left(\frac{\mathcal{J}_{t-h, t}^{2}-\overline{\mathcal{J}}_{-h}^{2}}{T^{3 / 2}}\right) \\ \frac{1}{T} \sum_{t=1}^{T-2 h+1}\left(\frac{C_{t-h, t}-\overline{C_{-h}}}{T^{3 / 2}}\right)\left(\frac{\mathcal{J}_{t-h, t}^{2}-\overline{\mathcal{J}_{-h}^{2}}}{T^{3 / 2}}\right) & \frac{1}{T} \sum_{t=1}^{T-2 h+1}\left(\frac{\mathcal{J}_{t-h, t}^{2}-\overline{\mathcal{J}_{-h}^{2}}}{T^{3 / 2}}\right)^{2}\end{array}\right]^{-1}$

where

$S^{2}=\frac{1}{T-2 h+1}\left[\sum_{t=1}^{T-2 h+1}\left(\left(R_{t, t+h}-\overline{R_{h}}\right)-\widehat{b}_{h}\left(C_{t-h, t}-\overline{C_{-h}}\right)-\widehat{\gamma}_{h}\left(\mathcal{J}_{t-h, t}^{2}-\overline{\mathcal{J}_{-h}^{2}}\right)\right)^{2}\right]$.

Thus: 


$$
\frac{S^{2}}{T}=\frac{T}{T-2 h+1}\left[\begin{array}{c}
\frac{1}{T} \sum_{t=1}^{T-2 h+1}\left(\frac{R_{t, t+h}-\overline{R_{h}}}{T^{1 / 2}}\right)^{2}+\left(T \widehat{b}_{h}\right)^{2} \frac{1}{T} \sum_{t=1}^{T-2 h+1}\left(\frac{C_{t-h, t}-\overline{C_{-h}}}{T^{3 / 2}}\right)^{2}+\left(T \widehat{\gamma}_{h}\right)^{2} \frac{1}{T} \sum_{t=1}^{T-2 h+1}\left(\frac{\mathcal{J}_{t-h, t}^{2}-\overline{\mathcal{J}_{-h}^{2}}}{T^{3 / 2}}\right)^{2} \\
+2 T \widehat{b}_{h} T \widehat{\gamma}_{h} \frac{1}{T} \sum_{t=1}^{T-2 h+1}\left(\frac{C_{t-h, t}-\overline{C_{-h}}}{T^{3 / 2}}\right)\left(\frac{\mathcal{J}_{t-h, t}^{2}-\overline{\mathcal{J}_{-h}^{2}}}{T^{3 / 2}}\right)-2 T \widehat{b}_{h} \frac{1}{T} \sum_{t=1}^{T-2 h+1}\left(\frac{C_{t-h, t}-\overline{C_{-h}}}{T^{3 / 2}}\right)\left(\frac{R_{t, t+h}-\overline{R_{h}}}{T^{1 / 2}}\right) \\
-2 T \widehat{\gamma}_{h} \frac{1}{T} \sum_{t=1}^{T-2 h+1}\left(\frac{\mathcal{J}_{t-h, t}^{2}-\overline{\mathcal{J}_{-h}^{2}}}{T^{3 / 2}}\right)\left(\frac{R_{t, t+h}-\overline{R_{h}}}{T^{1 / 2}}\right)
\end{array}\right] .
$$

Combining the Functional Central Limit Theorem (FCLT) and the Continuous Mapping Theorem (CMT), it follows that:

$$
\frac{S^{2}}{T} \stackrel{\mathcal{L}}{\longrightarrow} \frac{\sigma_{e}^{2}}{1-2 \lambda}\left[\begin{array}{c}
\int_{\lambda}^{1-\lambda} \bar{W}^{2}(s, \lambda) d s \\
-\bar{\Delta}^{-1} \int_{\lambda}^{1-\lambda} \bar{J}_{c_{1}}^{2}(s,-\lambda) d s\left(\int_{\lambda}^{1-\lambda} \bar{W}(s, \lambda) \bar{J}_{c_{2}}(s,-\lambda) d s\right)^{2} \\
-\bar{\Delta}^{-1} \int_{\lambda}^{1-\lambda} \bar{J}_{c_{2}}^{2}(s,-\lambda) d s\left(\int_{\lambda}^{1-\lambda} \bar{W}(s, \lambda) \bar{J}_{c_{1}}(s,-\lambda) d s\right)^{2} \\
+2 \bar{\Delta}^{-1} \int_{\lambda}^{1-\lambda} \bar{W}(s, \lambda) \bar{J}_{c_{1}}(s,-\lambda) d s \int_{\lambda}^{1-\lambda} \bar{J}_{c_{1}}(s,-\lambda) \bar{J}_{c_{2}}(s,-\lambda) d s \int_{\lambda}^{1-\lambda} \bar{W}(s, \lambda) \bar{J}_{c_{2}}(s,-\lambda) d s
\end{array}\right] .
$$

That is, $\frac{S^{2}}{T} \stackrel{\mathcal{L}}{\longrightarrow} \frac{\sigma_{e}^{2}}{1-2 \lambda} \bar{\Omega}$, where

$\bar{\Delta}=\int_{\lambda}^{1-\lambda} \bar{J}_{c_{1}}^{2}(s,-\lambda) d s \int_{\lambda}^{1-\lambda} \bar{J}_{c_{2}}^{2}(s,-\lambda) d s-\left(\int_{\lambda}^{1-\lambda} \bar{J}_{c_{1}}(s,-\lambda) \bar{J}_{c_{2}}(s,-\lambda) d s\right)^{2}$,

and:

$$
\bar{\Omega}=\left[\begin{array}{c}
\int_{\lambda}^{1-\lambda} \bar{W}^{2}(s, \lambda) d s \\
-\bar{\Delta}^{-1} \int_{\lambda}^{1-\lambda} \bar{J}_{c_{1}}^{2}(s,-\lambda) d s\left(\int_{\lambda}^{1-\lambda} \bar{W}(s, \lambda) \bar{J}_{c_{2}}(s,-\lambda) d s\right)^{2} \\
-\bar{\Delta}^{-1} \int_{\lambda}^{1-\lambda} \bar{J}_{c_{2}}^{2}(s,-\lambda) d s\left(\int_{\lambda}^{1-\lambda} \bar{W}(s, \lambda) \bar{J}_{c_{1}}(s,-\lambda) d s\right)^{2} \\
+2 \bar{\Delta}^{-1} \int_{\lambda}^{1-\lambda} \bar{W}(s, \lambda) \bar{J}_{c_{1}}(s,-\lambda) d s \int_{\lambda}^{1-\lambda} \bar{J}_{c_{1}}(s,-\lambda) \bar{J}_{c_{2}}(s,-\lambda) d s \int_{\lambda}^{1-\lambda} \bar{W}(s, \lambda) \bar{J}_{c_{2}}(s,-\lambda) d s
\end{array}\right] .
$$

Finally:

$$
T^{3} \operatorname{Var}\left(\begin{array}{c}
\widehat{b}_{h} \\
\widehat{\gamma}_{h}
\end{array}\right) \stackrel{\mathcal{L}}{\longrightarrow} \frac{\sigma_{e}^{2} \bar{\Omega}}{1-2 \lambda}\left[\begin{array}{cc}
\sigma_{u^{C}}^{2} \int_{\lambda}^{1-\lambda} \bar{J}_{c_{1}}^{2}(s,-\lambda) d s & \sigma_{u^{C}} \sigma_{u^{\mathcal{J}^{2}}} \int_{\lambda}^{1-\lambda} \bar{J}_{c_{1}}(s,-\lambda) \bar{J}_{c_{2}}(s,-\lambda) d s \\
\sigma_{u^{C}} \sigma_{u^{\mathcal{J}^{2}}} \int_{\lambda}^{1-\lambda} \bar{J}_{c_{1}}(s,-\lambda) \bar{J}_{c_{2}}(s,-\lambda) d s & \sigma_{u \mathcal{J}^{2}}^{2} \int_{\lambda}^{1-\lambda} \bar{J}_{c_{2}}^{2}(s,-\lambda) d s
\end{array}\right]^{-1},
$$

or equivalently:

$$
T^{3} \operatorname{Var}\left(\begin{array}{c}
\widehat{b}_{h} \\
\widehat{\gamma}_{h}
\end{array}\right) \stackrel{\mathcal{L}}{\longrightarrow} \frac{\bar{\Omega}}{(1-2 \lambda) \bar{\Delta}}\left[\begin{array}{cc}
\frac{\sigma_{e}^{2}}{\sigma_{u}^{2}} \int_{\lambda}^{1-\lambda} \bar{J}_{c_{2}}^{2}(s,-\lambda) d s & \frac{-\sigma_{e}^{2}}{\sigma_{u} \sigma_{u} \mathcal{J}^{2}} \int_{\lambda}^{1-\lambda} \bar{J}_{c_{1}}(s,-\lambda) \bar{J}_{c_{2}}(s,-\lambda) d s \\
\frac{-\sigma_{e}^{2}}{\sigma_{u} \sigma_{u} \mathcal{J}^{2}} \int_{\lambda}^{1-\lambda} \bar{J}_{c_{1}}(s,-\lambda) \bar{J}_{c_{2}}(s,-\lambda) d s & \frac{\sigma_{e}^{2}}{\sigma_{u}^{2}} \int_{\lambda}^{1-\lambda} \bar{J}_{c_{1}}^{2}(s,-\lambda) d s
\end{array}\right] .
$$

\section{B2: Proof of Proposition 2}

The proof for Proposition 2 follows identical steps. However, since we consider zero-intercept regressions, $\bar{W}(s, \lambda)$ and $\bar{J}_{c}(s,-\lambda)$ are replaced with their uncentered versions $W(s, \lambda)$ and $J_{c}(s,-\lambda)$. 


\section{B3: $\quad$ Proof of Proposition 3}

1. $T \widehat{\beta}_{h_{l}}=\frac{\frac{1}{T} \sum_{t=1}^{T-2 h_{l}+1}\left(\frac{C_{t-h_{l}, t}-\overline{C_{-h_{l}}}}{T^{3 / 2}}\right)\left(\frac{R_{t, t+h_{l}}-\overline{R_{h_{l}}}}{T^{1 / 2}}\right)}{\frac{1}{T} \sum_{t=1}^{T-2 h_{l}+1}\left(\frac{C_{t-h_{l}, t}-\overline{C_{-h_{l}}}}{T^{3 / 2}}\right)^{2}} \stackrel{\mathcal{L}}{\longrightarrow} \frac{\sigma_{\varepsilon}}{\sigma_{u}} \frac{\int_{\lambda_{i}}^{1-\lambda_{l}} \bar{W}\left(s, \lambda_{l}\right) \bar{J}_{c}\left(s,-\lambda_{l}\right) d s}{\int_{\lambda_{l}}^{1-\lambda} \bar{J}_{c}^{2}\left(s,-\lambda_{l}\right) d s}$, for $l=i, j$,

2. $T^{3} \operatorname{Var}\left(\begin{array}{c}\widehat{\beta}_{h_{i}} \\ \widehat{\beta}_{h_{j}}\end{array}\right)=T^{3}\left(\begin{array}{cc}\operatorname{Var}\left(\widehat{\beta}_{h_{i}}\right) & \operatorname{Cov}\left(\widehat{\beta}_{h_{i}}, \widehat{\beta}_{h_{j}}\right) \\ \operatorname{Cov}\left(\widehat{\beta}_{h_{i}}, \widehat{\beta}_{h_{j}}\right) & \operatorname{Var}\left(\widehat{\beta}_{h_{j}}\right)\end{array}\right)$

$$
T^{3} \operatorname{Var}\left(\widehat{\beta}_{h_{l}}\right)=\frac{S_{l}^{2}}{T}\left(\frac{1}{T} \sum_{t=1}^{T-2 h_{l}+1}\left(\frac{C_{t-h_{l}, t}-\overline{C_{-h_{l}}}}{T^{3 / 2}}\right)^{2}\right)^{-1}, \text { for } l=i, j,
$$

where

$$
S_{l}^{2}=\frac{1}{T-2 h_{l}+1}\left[\sum_{t=1}^{T-2 h_{l}+1}\left(R_{t, t+h_{l}}-\overline{R_{h_{l}}}\right)^{2}-\widehat{\beta}_{h_{l}}^{2} \sum_{t=1}^{T-2 h_{l}+1}\left(C_{t-h_{l}, t}-\overline{C_{-h_{l}}}\right)^{2}\right] .
$$

Moreover:

$T^{3} \operatorname{Cov}\left(\widehat{\beta}_{h_{i}}, \widehat{\beta}_{h_{j}}\right)=\frac{S_{i j}}{T}\left[\frac{\left(\frac{1}{T} \sum_{t=1}^{T-2 h_{(i \vee j)}+1}\left(\frac{C_{t-h_{i}, t}-\overline{C_{-h_{i}}}}{T^{3 / 2}}\right)\left(\frac{C_{t-h_{j}, t}-\overline{C_{-h_{j}}}}{T^{3 / 2}}\right)\right)}{\left(\frac{1}{T} \sum_{t=1}^{T-2 h_{i}+1}\left(\frac{C_{t-h_{i}, t}-\overline{C_{-h_{i}}}}{T^{3 / 2}}\right)^{2}\right)\left(\frac{1}{T} \sum_{t=1}^{T-2 h_{j}+1}\left(\frac{C_{t-h_{j}, t}-\overline{C_{-h_{j}}}}{T^{3 / 2}}\right)^{2}\right)}\right]$,

where: $S_{i j}=\frac{1}{T-2 h_{(i \vee j)}+1}\left[\begin{array}{c}\sum_{t=1}^{T-2 h_{(i \vee j)}+1}\left(R_{t, t+h_{i}}-\overline{R_{h_{i}}}\right)\left(R_{t, t+h_{j}}-\overline{R_{h_{j}}}\right) \\ -\widehat{\beta}_{h_{i}} \widehat{\beta}_{h_{j}} \sum_{t=1}^{T-2 h_{(i \vee j)}+1}\left(C_{t-h_{i}, t}-\overline{C_{-h_{i}}}\right)\left(C_{t-h_{j}, t}-\overline{C_{-h_{j}}}\right)\end{array}\right]$ represents the covariance between the two regressions error terms and $h_{(i \vee j)} \equiv \max \left(h_{i}, h_{j}\right)$. Note that:

$\overline{R_{h_{l}}}=\frac{1}{T-2 h_{l}+1} \sum_{t=1}^{T-2 h_{l}+1} R_{t, t+h_{l}}$ and $\overline{C_{-h_{l}}}=\frac{1}{T-2 h_{l}+1} \sum_{t=1}^{T-2 h_{l}+1} C_{t-h_{l}, t}$, for $l=i, j$.

Again using the FCLT and the Continuous Mapping Theorem (CMT) it follows that:

$$
\begin{gathered}
\frac{S_{l}^{2}}{T}\left(\frac{1}{T} \sum_{t=1}^{T-2 h_{l}+1}\left(\frac{C_{t-h_{l}, t}-\overline{C_{-h_{l}}}}{T^{3 / 2}}\right)^{2}\right)^{-1}=\frac{T}{T-2 h_{l}+1} \frac{\left[\frac{1}{T} \sum_{t=1}^{T-2 h_{l}+1}\left(\frac{R_{t, t+h_{l}}-\overline{R_{h_{l}}}}{T^{1 / 2}}\right)^{2}-\left(T \widehat{\beta}_{h_{l}}\right)^{2} \frac{1}{T} \sum_{t=1}^{T-2 h_{l}+1}\left(\frac{C_{t-h_{l}, t}-\overline{C_{-h_{l}}}}{T^{3 / 2}}\right)^{2}\right]}{\frac{1}{T} \sum_{t=1}^{T-2 h_{l}+1}\left(\frac{C_{t-h_{l}, t}-\overline{C_{-h_{l}}}}{T^{3 / 2}}\right)^{2}} \\
\stackrel{\mathcal{L}}{\longrightarrow} \frac{\sigma_{\varepsilon}^{2}}{\sigma_{u}^{2}} \frac{1}{1-2 \lambda_{l}} \frac{\left[\int_{\lambda_{l}}^{1-\lambda_{l}} \bar{W}^{2}\left(s, \lambda_{l}\right) d s \int_{\lambda_{l}}^{1-\lambda_{l}} \bar{J}_{c}^{2}\left(s,-\lambda_{l}\right) d s-\left(\int_{\lambda_{l}}^{1-\lambda_{l}} \bar{W}\left(s, \lambda_{l}\right) \bar{J}_{c}\left(s,-\lambda_{l}\right) d s\right)^{2}\right]}{\left(\int_{\lambda_{l}}^{1-\lambda_{l}} \bar{J}_{c}^{2}\left(s,-\lambda_{l}\right) d s\right)^{2}}, l=i, j
\end{gathered}
$$

and

$$
\frac{S_{i j}}{T}\left[\frac{\left(\frac{1}{T} \sum_{t=1}^{T-2 h_{(i \vee j)}+1}\left(\frac{C_{t-h_{i}, t}-\overline{C_{-h_{i}}}}{T^{3 / 2}}\right)\left(\frac{C_{t-h_{j}, t}-\overline{C_{-h_{j}}}}{T^{3 / 2}}\right)\right)}{\left(\frac{1}{T} \sum_{t=1}^{T-2 h_{i}+1}\left(\frac{C_{t-h_{i}, t}-\overline{C_{-h_{i}}}}{T^{3 / 2}}\right)^{2}\right)\left(\frac{1}{T} \sum_{t=1}^{T-2 h_{j}+1}\left(\frac{C_{t-h_{j}, t}-\overline{C_{-h_{j}}}}{T^{3 / 2}}\right)^{2}\right)}\right]
$$




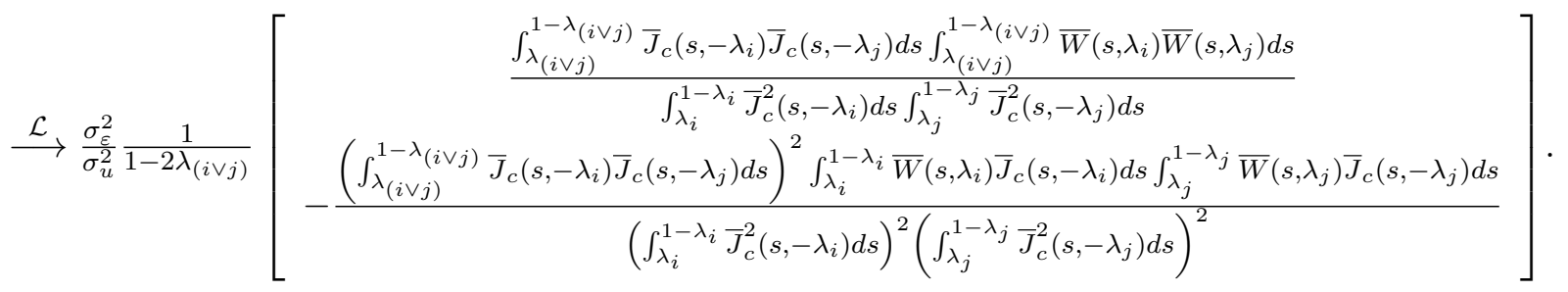

Hence, $T^{3} \operatorname{Var}\left(\widehat{\beta}_{h_{l}}\right) \stackrel{\mathcal{L}}{\longrightarrow} \overline{\operatorname{Avar}}\left(\widehat{\beta}_{h_{l}}\right)$, where

$\overline{\operatorname{Avar}}\left(\widehat{\beta}_{h_{l}}\right)=\frac{\sigma_{\varepsilon}^{2}}{\sigma_{u}^{2}} \frac{1}{1-2 \lambda_{l}} \frac{\left[\int_{\lambda_{l}}^{1-\lambda_{l}} \bar{W}^{2}\left(s, \lambda_{l}\right) d s \int_{\lambda_{l}}^{1-\lambda_{l}} \bar{J}_{c}^{2}\left(s,-\lambda_{l}\right) d s-\left(\int_{\lambda_{l}}^{1-\lambda_{l}} \bar{W}\left(s, \lambda_{l}\right) \bar{J}_{c}\left(s,-\lambda_{l}\right) d s\right)^{2}\right]}{\left(\int_{\lambda_{l}}^{1-\lambda_{l}} \bar{J}_{c}^{2}\left(s,-\lambda_{l}\right) d s\right)^{2}}$, for $l=i, j$.

Finally, $T^{3} \operatorname{Cov}\left(\widehat{\beta}_{h_{i}}, \widehat{\beta}_{h_{j}}\right) \stackrel{\mathcal{L}}{\longrightarrow} \overline{\operatorname{Acov}}\left(\widehat{\beta}_{h_{i}}, \widehat{\beta}_{h_{j}}\right)$, where

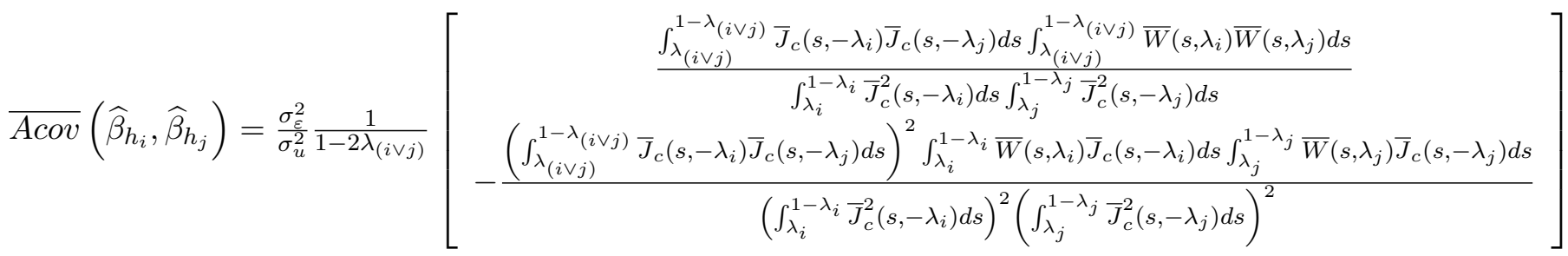

and $\lambda_{(i \vee j)} \equiv \max \left(\lambda_{i}, \lambda_{j}\right)$.

\section{B4: Proof of Proposition 4}

The proof for Proposition 4 is similar except that $\bar{W}(s, \lambda)$ and $\bar{J}_{c}(s,-\lambda)$ are replaced with their uncentered versions $W(s, \lambda)$ and $J_{c}(s,-\lambda)$.

\section{B5: Proof of Equation (15)}

The rescaled Wald statistic is used to test the hypothesis $\mathcal{R}\left[\begin{array}{c}\widehat{\beta}_{h_{i}} \\ \widehat{\beta}_{h_{j}}\end{array}\right]=0$ where $\mathcal{R}=\left[\begin{array}{ll}-1 & 1\end{array}\right]$.

Recall that:

$$
T^{-1} \chi_{\left(\widehat{\beta}_{h_{j}=\left\lfloor\lambda_{j} T\right\rfloor}^{2}-\widehat{\beta}_{h_{i}=\left\lfloor\lambda_{i} T\right\rfloor}\right)}^{2}=\frac{\left[T\left(\widehat{\beta}_{h_{j}}-\widehat{\beta}_{h_{i}}-0\right)\right]^{2}}{T^{3}\left[\operatorname{Var}\left(\widehat{\beta}_{h_{j}}\right)+\operatorname{Var}\left(\widehat{\beta}_{h_{i}}\right)-2 \operatorname{Cov}\left(\widehat{\beta}_{h_{i}}, \widehat{\beta}_{h_{j}}\right)\right]} .
$$

By FCLT and CMT, we have: $\left[T\left(\widehat{\beta}_{h_{j}}-\widehat{\beta}_{h_{i}}-0\right)\right]^{2} \stackrel{\mathcal{L}}{\longrightarrow} \bar{N}$, where

$$
\bar{N}=\frac{\sigma_{\varepsilon}^{2}}{\sigma_{u}^{2}}\left[\frac{\int_{\lambda_{j}}^{1-\lambda_{j}} \bar{W}\left(s, \lambda_{j}\right) \bar{J}_{c}\left(s,-\lambda_{j}\right) d s}{\int_{\lambda_{j}}^{1-\lambda_{j}} \bar{J}_{c}^{2}\left(s,-\lambda_{j}\right) d s}-\frac{\int_{\lambda_{i}}^{1-\lambda_{i}} \bar{W}\left(s, \lambda_{i}\right) \bar{J}_{c}\left(s,-\lambda_{i}\right) d s}{\int_{\lambda_{i}}^{1-\lambda_{i}} \bar{J}_{c}^{2}\left(s,-\lambda_{i}\right) d s}\right]^{2} .
$$


Now focus on the denominator; from the same argument as before, we get:

$$
T^{3}\left[\operatorname{Var}\left(\widehat{\beta}_{h_{j}}\right)+\operatorname{Var}\left(\widehat{\beta}_{h_{i}}\right)-2 \operatorname{Cov}\left(\widehat{\beta}_{h_{i}}, \widehat{\beta}_{h_{j}}\right)\right] \stackrel{\mathcal{L}}{\longrightarrow} \bar{D},
$$

where $\bar{D}=\overline{\operatorname{Avar}}\left(\widehat{\beta}_{h_{i}}\right)+\overline{\operatorname{Avar}}\left(\widehat{\beta}_{h_{j}}\right)-2 \overline{\operatorname{Acov}}\left(\widehat{\beta}_{h_{i}}, \widehat{\beta}_{h_{j}}\right)$. Combining the numerator and the denominator yields Eqn. (15). Eqn. (16) obtains in the same way, but $\bar{W}(s, \lambda)$ and $\bar{J}_{c}(s,-\lambda)$ are replaced with their uncentered versions $W(s, \lambda)$ and $J_{c}(s,-\lambda)$. 